\title{
Peering through the veil: near-infrared photometry and extinction for the Galactic nuclear star cluster
}

\section{Accurate near infrared $H, K s$, and $L^{\prime}$ photometry and the near-infrared extinction-law toward the central parsec of the Galaxy ${ }^{\star}$}

\author{
R. Schödel ${ }^{1}$, F. Najarro ${ }^{2}$, K. Muzic ${ }^{3}$, and A. Eckart ${ }^{3}$ \\ ${ }^{1}$ Instituto de Astrofísica de Andalucía - CSIC, Glorieta de la Astronomía S/N, 18008 Granada, Spain \\ e-mail: rainer@iaa.es \\ 2 Centro de Astrobiología (CSIC/INTA), Instituto Nacional de Técnica Aeroespacial, Ctra de Torrejón a Ajalvir, km 4, \\ 28850 Torrejón de Ardoz, Madrid, Spain \\ e-mail: najarro@damir.iem.cisc.es \\ 3 I. Physikalisches Institut, Universität zu Köln, Zülpicher Strasse 77, 50937 Köln, Germany \\ e-mail: [muzic; eckart]@ph1.uni-koeln.de
}

Received 26 August 2009 / Accepted 2 December 2009

ABSTRACT

\begin{abstract}
Context. The nuclear star cluster of the Galaxy is an important template for understanding its extragalactic counterparts, which can currently not be resolved into individual stars. Important drawbacks of observations of the Galactic center are, however, the presence of strong and spatially highly variable interstellar extinction and extreme crowding of the sources, which makes the use of adaptive optics techniques necessary. Both points pose serious obstacles to precise photometry that is needed for analyzing the stellar population. Aims. The aims of this work are to provide accurate photometry in multiple near-infrared broadband filters, to determine the powerlaw index of the extinction-law toward the central parsec of the Galaxy, to provide measurements of the absolute extinction toward the Galactic center, and finally to measure the spatial variability of extinction on arcsecond scales.

Methods. We use observations of the central parsec of the Milky Way that were obtained with the near-infrared camera and adaptive optics system NAOS/CONICA at the ESO VLT unit telescope 4. The photometric method takes into account anisoplanatic effects and limits the corresponding systematic uncertainties to $\lesssim 2 \%$. Absolute values for the extinction in the $H, K$ s, and $L^{\prime}$-bands as well as of the power-law indices of the $H$ to $K \mathrm{~s}$ and $K \mathrm{~s}$ to $L^{\prime}$ extinction-laws are measured based on the well-known properties of red clump stars. Extinction maps are derived based on $H-K \mathrm{~s}$ and $K \mathrm{~s}-L^{\prime}$ colors.

Results. We present $K$ s-band photometry for $\sim 7700$ stars, and additionally photometry for stars detected in the $H$ and/or $L^{\prime}$-bands. From a number of recently published values we compute a mean distance of the Galactic center of $R_{0}=8.03 \pm 0.15 \mathrm{kpc}$, which has an uncertainty of just $2 \%$. Based on this $R_{0}$ and on the RC method, we derive absolute mean extinction values toward the central parsec of the Galaxy of $A_{H}=4.48 \pm 0.13 \mathrm{mag}, A_{K \mathrm{~s}}=2.54 \pm 0.12 \mathrm{mag}$, and $A_{L^{\prime}}=1.27 \pm 0.18 \mathrm{mag}$. We estimate values of the power-law indices of the extinction-law of $\alpha_{H-K \mathrm{~s}}=2.21 \pm 0.24$ and $\alpha_{K \mathrm{~s}-L^{\prime}}=1.34 \pm 0.29$. A $K \mathrm{~s}$-band extinction map for the Galactic center is computed based on this extinction law and on stellar $H-K$ s colors. Both its statistical and systematic uncertainties are estimated to be $<10 \%$. Extinction in this map derived from stellar color excesses is found to vary on arcsecond scales, with a mean value of $A_{K \mathrm{~s}}=2.74 \pm 0.30 \mathrm{mag}$. Mean extinction values in a circular region with $0.5^{\prime \prime}$ radius centered on Sagittarius $\mathrm{A}^{*}$ are $A_{H, \mathrm{SgrA} *}=4.35 \pm 0.12, A_{K \mathrm{~s}, \mathrm{SgrA} *}=2.46 \pm 0.03$, and $A_{L^{\prime}, \mathrm{SgrA} *}=1.23 \pm 0.08$.
\end{abstract}

Key words. Galaxy: nucleus - dust, extinction - Galaxy: center - stars: horizontal-branch

\section{Introduction}

Nuclear star clusters (NSCs) are located at the photometric and dynamical centers of almost all spiral galaxies. Their detailed study has only become possible via HST observations in the 1990 s, but they are of great interest because they are the densest and most massive clusters in their host galaxies. Frequently they contain supermassive black holes at their centers. They show signs of recurring star formation, and the discovery of rotation of the NSCs of NGC 4244 (Seth et al. 2008) and of the Milky Way (Trippe et al. 2008; Schödel et al. 2009) indicates that NSCs may at least partially form by accretion of material from their

^ Full Table A.2 is only available in electronic form at the CDS via anonymous ftp to

cdsarc.u-strasbg.fr $(130.79 .128 .5)$ or via

http://cdsweb.u-strasbg.fr/cgi-bin/qcat?J/A+A/511/A18

The extinction map shown in the top left of Fig. 6 can be obtained in electronic form at the same site. host galaxy. A concise review of the properties of NSCs and hypotheses on their formation is given in Böker (2008).

Studying the NSC at the Galactic center (GC) is of great importance for the understanding of NSCs in general because in the next decades it will remain impossible to resolve any but the brightest individual stars in extragalactic NSCs, even with $50 \mathrm{~m}$-class telescopes. A further reason for the importance of the GC NSC is that the existence of the supermassive black hole, Sagittarius A*, at the GC is well established and the mass of this black hole has been determined with high precision (e.g., Ghez et al. 2008; Gillessen et al. 2009). Strong interest in the stellar population at the GC was raised by spectroscopic observations, which have revealed the existence of numerous massive, young stars within $0.5 \mathrm{pc}$ of Sagittarius A*. Those young stars are witnesses of a starburst event that occurred about 4 million years ago. The young stars are arranged in a non-isotropic pattern and at least $50 \%$ of them are located in a rotating disk (see Bartko et al. 2009; Lu et al. 2009). 
Unfortunately, there are two important drawbacks in observational studies of the Milky Way's NSC: extreme crowding and high extinction. While the great progress made in adaptive optics techniques at 8-10 m-class telescopes in the past years has been highly successful in reducing the problem of crowding, it nevertheless remains a serious issue. Even with instruments such as NACO at the ESO VLT, strong crowding limits the point-source sensitivity within $\sim 0.5 \mathrm{pc}$ of Sagittarius A* so significantly that only stars more massive than $\sim 2 M_{\odot}$ can be detected in this region. This means that we can still detect only of the order $10 \%$ of the stars in the NSC (Schödel et al. 2007a). Additionally, it is notoriously difficult to extract accurate photometric measurements from $\mathrm{AO}$ observations of crowded fields, particularly when the field-of-view exceeds the size of the isoplanatic angle.

As concerns extinction, we can consider ourselves lucky that we can observe the GC at all. Within 2-3 pc of Sagittarius A* there exist plenty of clouds that are almost or completely opaque even in the near-infrared (for example, see Fig. 1 in Schödel et al. 2007a). Average extinction toward the central parsec in the $K$-band reaches $A_{K} \approx 3$. As concerns extinction at visual wavelengths, Rieke \& Lebofsky (1985) found $A_{V} / A_{K} \sim 9$, but more recent studies indicate higher values of $A_{V} / A_{K} \sim 16$ (Nishiyama et al. 2008), or even $A_{V} / A_{K} \sim 29$ (Gosling et al. 2009). Therefore, the stellar population at the GC becomes observable only at near-infrared wavelengths. Only the brightest stars can be seen in the $J$-band. Reasonable $\mathrm{S} / \mathrm{N}$ on the stars is only reached in the $H, K$, and $L$ bands. This restriction to a tight wavelength window, where most stars show only small color indices, combined with the fact that the extinction toward the GC is not only high, but also variable on small spatial scales of a few arcseconds (Scoville et al. 2003; Gosling et al. 2006; Schödel et al. 2007a), makes photometric studies of the stellar population at the GC extremely difficult.

Although adaptive optics assisted integral field spectroscopy has resulted in the classification of hundreds of stars within $\sim 0.5 \mathrm{pc}$ of Sagittarius A* (e.g., Maness et al. 2007; Paumard et al. 2006; Do et al. 2009a), this technique is extremely time consuming in both observations and data analysis. In order to efficiently probe large fields in the Galactic center for the presence of young, massive stars, it is therefore desirable to improve the photometric observations in a way that enables us to at least crudely distinguish whether a star in the GC is of early or of late spectral type. How this can be done in spite of the difficulties described above has been demonstrated recently by Buchholz et al. (2009). They used intermediate band filters across the K-band, calibrated photometry locally with the help of red clump stars, and used the $\mathrm{CO}$ bandhead absorption feature of late-type stars as a distinguishing criterion.

Accurate knowledge of extinction has impact in many different aspects of GC research: the stellar surface number density is not only a function of distance from Sagittarius A* but is also dependent on local extinction (Schödel et al. 2007a); extinction is an important ingredient for modeling stellar atmospheres (e.g., Najarro et al. 1997; Martins et al. 2007); extinction in the infrared is also important for estimating the intrinsic brightness of the near-infrared emission from the accretion flow or outflow related to Sagittarius A* (e.g., Genzel et al. 2003; Eckart et al. 2006; Do et al. 2009b). The mentioned examples show that one must know not only the absolute value of extinction, but also how it changes as a function of wavelength and how it varies on spatial scales as small as one to a few arcseconds.

Until just about a decade ago, the extinction law from nearto mid-infrared wavelengths $(\sim 1-8 \mu \mathrm{m})$ was thought to be described well by a "universal" power-law, $A_{\lambda} \propto \lambda^{-\alpha}$, with a
Table 1. Details of the imaging observations used in this work.

\begin{tabular}{cccccc}
\hline \hline Date & $\begin{array}{c}\lambda_{\text {central }}[\mu \mathrm{m}] \\
{[\mu \mathrm{m}]}\end{array}$ & $\begin{array}{c}\Delta \lambda \\
{[\mu}\end{array}$ & NDIT $^{b}$ & $\begin{array}{c}\text { DIT }^{c} \\
{[\mathrm{~s}]}\end{array}$ \\
\hline 29 April 2006 & 1.66 & 0.33 & 32 & 28 & 2 \\
29 April 2006 & 2.18 & 0.35 & 32 & 28 & 2 \\
26 May 2008 & 3.80 & 0.62 & 202 & 150 & 0.2 \\
\hline
\end{tabular}

Notes. ${ }^{(a)}$ Number of (dithered) exposures. ${ }^{(b)}$ Number of integrations that were averaged on-line by the read-out electronics. ${ }^{(c)}$ Detector integration time. The total integration time of each observation amounts to $N \times$ NDIT $\times$ DIT .

power-law index of $\alpha \approx 1.75$ (e.g., Draine 1989). First doubts were shed on this model by spectroscopic observations of the GC with ISO SWS that showed clear deviations from this model at wavelengths $\gtrsim 3 \mu \mathrm{m}$ (Lutz et al. 1996). Subsequent studies have confirmed that the near- to mid-infrared extinction-law is more complex than a simple power-law. While it seems well established by now that a simple power-law is an adequate description of the wavelength dependence of extinction in the near-infrared, albeit with a stronger wavelength dependence as previously thought, the situation is less clear longward of $\sim 3 \mu \mathrm{m}$. Recent investigation shows that the extinction law decreases much less rapidly at $\lambda>3 \mu \mathrm{m}$ than suggestd by earlier estimates and may actually be almost constant at 5-10 $\mu \mathrm{m}$ (see Nishiyama et al. 2009 , for an extensive discussion of these points). Taking the problem even a step further, in a recent study of extinction toward the nuclear bulge Gosling et al. (2009) find that the index of the power-law extinction-law can change on spatial scales as small as 5 arcsec. This signifies that we may be forced to abandon the concept of a universal extinction-law.

It is evident that the topic of extinction toward the central parsec of the Galaxy needs to be re-visited. Extinction maps for the central $0.5-1 \mathrm{pc}$ were recently presented by Schödel et al. (2007a) and Buchholz et al. (2009). However, these papers were not mainly focused on extinction and the extinction maps were rather side-products. Both papers used the old $A_{\lambda} \propto \lambda^{-1.75}$ extinction-law (Draine 1989). Neither the extinction law nor absolute extinction were examined in depth. Also, the absolute photometric calibration in our previous works was of limited accuracy because the PSF for the stars was either truncated (Schödel et al. 2007a) or because complex local calibration procedures had to be adopted (Buchholz et al. 2009).

The aim of this paper is to carry us a step forward in observational studies of the GC NSC by addressing two of the above described problems: accurate photometry with AO over a large FOV and accurate measurements of extinction. Schödel (2010) has recently presented a method for the photometric analysis of AO data with spatially variable PSF, but a very limited number of suitable PSF reference stars. Based on this method, in this paper we analyze imaging observations of the GC in the $H, K \mathrm{~s}$, and $L^{\prime}$ bands and provide accurate photometry (with both statistical and systematic uncertainties of the order $\sim 5 \%$ at $K \mathrm{~s}$ ) for several thousand sources observed in these bands within $\sim 1 \mathrm{pc}$ of Sagittarius A*. We determine both the absolute extinction and the extinction law at the various bands and provide a detailed extinction map (resolution of $1^{\prime \prime}-2^{\prime \prime}$ ) for the central parsec of the GC.

\section{Observations}

The observations used in this work were obtained with the near-infrared camera and adaptive optics (AO) system NAOS/CONICA (short NACO) at the ESO VLT unit 
telescope $4^{1}$. The $K \mathrm{~s} \approx 6.5-7.0$ supergiant IRS 7 was used to close the loop of the AO, using the NIR wavefront sensor. The sky background was measured on a largely empty patch of sky, a dark cloud about 400" north and 713" east of the target. Sky subtraction, bad pixel correction, and flat fielding were applied to the individual exposures. The NACO S27 camera, with a pixel scale of $0.02705^{\prime \prime} /$ pix, was used for both $H$ and $K$ s band observations. The field-of-view (FOV) of a single exposure is thus $28^{\prime \prime} \times 28^{\prime \prime}$. The observations were dithered by applying a rectangular dither pattern with the center of the dithered exposures positioned approximately at $\left(8.0^{\prime \prime},-2.6^{\prime \prime}\right),\left(-6.1^{\prime \prime},-2.7^{\prime \prime}\right)$, $\left(-6.1^{\prime \prime}, 11.2^{\prime \prime}\right)$, and $\left(8.1^{\prime \prime}, 11.3^{\prime \prime}\right)$ east and north of Sgr A*. In the text we refer to these four offsets as dither positions $1,2,3$, and 4 . The combined FOV of the observations is about $40^{\prime \prime} \times 40^{\prime \prime}$ and is offset to the north with respect to Sgr A* because the guide star IRS 7 is located about 5.6" north of Sgr A*.

Seeing in the optical ranged between $0.6^{\prime \prime}$ and $1.0^{\prime \prime}$ for both $H$ and $K$ s observations. According to the ESO Ambient Conditions Database ${ }^{2}$ sky transparency variations during the observations were below $1 \%$. The achieved Strehl ratio ranged between $\sim 20 \%(\sim 15 \%)$ near the guide star and $\sim 8 \%(\sim 8 \%)$ at $25^{\prime \prime}$ distance from the guide star in the $K \mathrm{~s}-(H$-) band. The Strehl ratio was estimated using the Strehl algorithm of the ESO eclipse software package (Devillard 1997) on PSFs extracted at various positions in the image. From the multiple measurements we estimate the $1 \sigma$ uncertainty of the measured Strehl ratio to $\sim 3 \%$. Table 1 summarizes the observations. The detector integration time (DIT) was set to $2.0 \mathrm{~s}$ in order to avoid saturation of the brightest stars. The central pixels of a small number of the brightest stars were saturated in $K \mathrm{~s}$. Their cores were repaired using nearby unsaturated stars and the PSF extraction routine of StarFinder. After 28 DITs, the instrument averaged the data to a single exposure (NDIT $=28$ ). In this way, 8 individual exposures were obtained per dither position. The exposures of each respective dither position were aligned (to compensate for small residual shifts) with the jitter algorithm of the ESO eclipse software package (Devillard 1997). We show the combined FOV of the $K$ s-band observations in Fig. 1 . Note that the photometry and astrometry in this work was done on the combined images of each dither position and not on the combined mosaic of all images (as shown in Fig. 1) in order to have a constant signal-tonoise ratio over the entire images. The $\sim 13.5^{\prime \prime} \times 13.5^{\prime \prime}$ overlap area between the four dither positions is indicated by the rectangle in Fig. 1.

Seeing in the optical ranged between $1.0^{\prime \prime}$ and $2.0^{\prime \prime}$ for the $L^{\prime}$ observations. The NACO L27 camera, with a pixel scale of $0.02719^{\prime \prime} /$ pix, was used for the $L^{\prime}$ observations. According to the ESO Ambient Conditions Database sky transparency variations during the observations were below $1 \%$. The observations were random jittered, with a jitter box width of $20^{\prime \prime}$. Offsets by $60^{\prime \prime}$ in random directions were alternated with observations of the target. The frames taken on the offsets frames, where the stellar density is lower, were used to construct sky frames. The 7 offset frames nearest in time to a given exposure on the target were median combined and subtracted as sky measurement. Flat fielding and bad pixel correction were applied. Finally, all exposures were combined with the jitter algorithm of the ESO

\footnotetext{
${ }^{1}$ Based on observations made with ESO Telescopes at the La Silla or Paranal Observatories under programmes ID 077.B-0014 and 081.B0648.

${ }^{2}$ http://archive.eso.org/asm/ambient-server? site=paranal
}

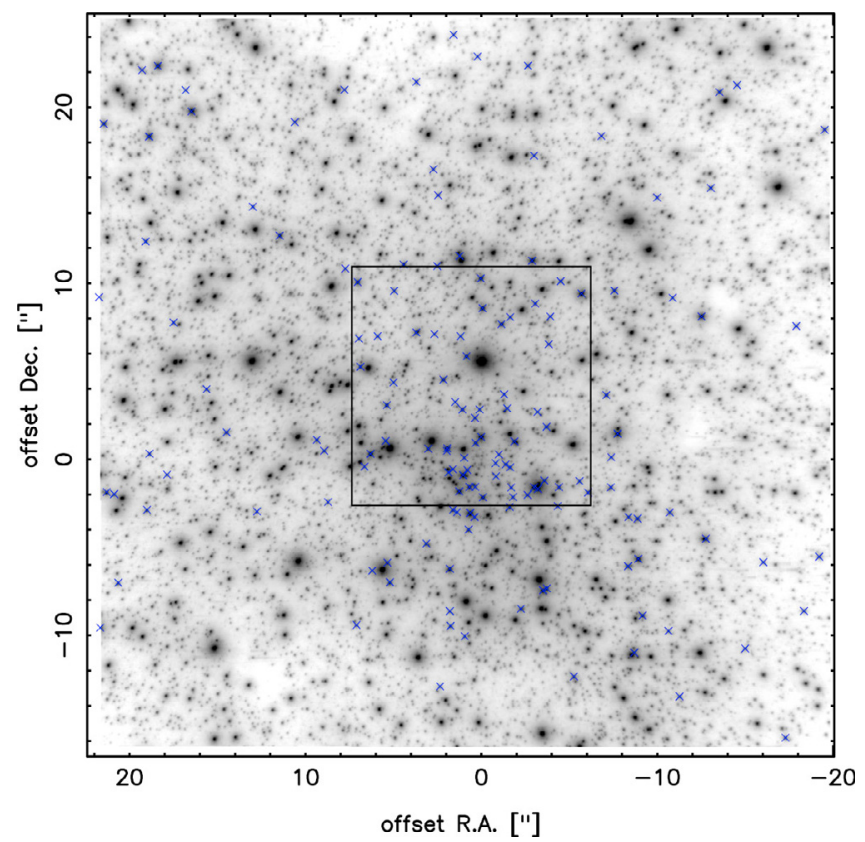

Fig. 1. Mosaic image of the $K$ s-band observations. North is up and east is to the left. Offsets are given in arcseconds from Sgr A*. The guide star, IRS 7, is the brightest star within the rectangle, about 5.5" north of Sgr A*. The black rectangle indicates the area of overlap between the 4 dither positions. Blue crosses mark early-type stars identified by their $H-K$ s colors with extinction/reddening correction applied (see Sect. 5 and Fig. 8).

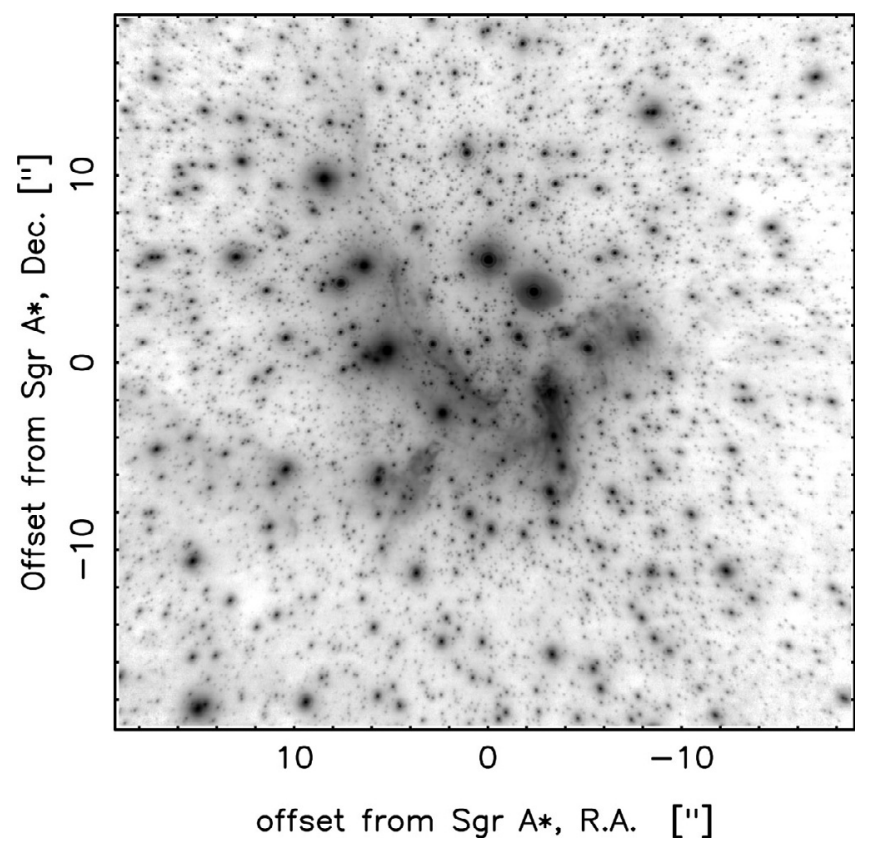

Fig. 2. Mosaic image of the $L^{\prime}$-band observations. North is up and east is to the left. Offsets are given in arcseconds from $\mathrm{Sgr} \mathrm{A}^{*}$.

eclipse software package. The resulting mosaic image is shown in Fig. 2.

\section{Photometry}

Due to the extreme source density at the GC aperture photometry cannot be applied accurately to GC imaging data. Therefore PSF fitting techniques must be used. In AO imaging 
anisoplanatic effects can lead to a systematic bias in the photometry of point sources when PSF fitting is used for estimating the flux of the stars. This bias depends on various factors such as distance from the guide star, observing wavelength, and atmospheric conditions at the time of the observations. In case of the $H$-band data used in this work the systematic error of the photometry can reach 0.25 mag across the FOV, if a single, non-variable PSF is used (see Schoedel, A\&A, 2009, accepted for publication). For example, anisoplanatic effects make stars at distances larger than the isoplanatic angle appear elongated, with their major axes pointing toward the guide star. This can be nicely seen in the image shown in Fig. 2 (note: usually anisoplanatic effects are stronger at shorter wavelengths, but in the observations used for this paper, it happens that those effects are stronger in the $L^{\prime}$-band than in the $K$ s-band image). Various methods for dealing with anisoplanatic effects have been suggested. A straightforward and effective method to deal with anisoplanatic effects is presented in a companion paper (Schoedel, A\&A, 2009, accepted for publication). It not only provides accurate point source photometry but also reliable estimation of the diffuse background emission. In brief, the method consists of two steps: (a) Wiener deconvolution, using a point spread function (PSF) extracted from the guide star (or bright stars near the guide star), in this case IRS 7; (b) photometry and astrometry on the deconvolved image via PSF fitting with the StarFinder (Diolaiti et al. 2000) program package. The image is partitioned into overlapping sub-frames that are smaller than the isoplanatic angle. Those sub-frames are analyzed independently. As shown in Schoedel (A\&A, 2009, accepted for publication) this method minimizes the systematic uncertainty of the point source photometry due to anisoplanatic effects to $\lesssim 0.02 \mathrm{mag}$ across the $28^{\prime \prime} \times 28^{\prime \prime}$ FOV of the images at each dither position.

After deconvolution with PSFs extracted from IRS 7, the $H$ and $K$ s-band images from the four dither positions were partitioned into $13 \times 13$ overlapping sub-frames of $256 \times 256$ pixel $^{2}$ $\left(\sim 6.9^{\prime \prime} \times 6.9^{\prime \prime}\right)$. The shifts between sub-frames were thus just 64 pixels in $x$ - and/or $y$-direction to assure large overlap between the sub-frames. We used the following StarFinder parameters: min_corr $=0.9$, thresh $=[5 ., 5$.$] , and back_box =30$. In order to avoid spurious detections, point source detection was also run on non-deconvolved images and only sources that were discovered in both the deconvolved and non-deconvolved images were included in the final list of detected stars. The images for the diffuse background and residuals were obtained by recombining the corresponding sub-frames. The method provides two kinds of uncertainties for the photometric and astrometric measurements: formal uncertainties, computed by the StarFinder algorithm from Gaussian and photon noise, and PSF uncertainties, resulting from incomplete knowledge of the local PSF. The latter is calculated by comparing multiple measurements of the stars because they are present in several sub-frames. For stars without multiple measurements we adopted a PSF uncertainty of $0.02 \mathrm{mag}$ for the $H$-band and $0.015 \mathrm{mag}$ for the $K$ s-band (see Fig. 3). The results of multiple measurements were averaged. Since deconvolution changes the noise properties of the image, the formal uncertainties computed by StarFinder are too small (for details see Schoedel, A\&A, 2009, accepted for publication). Monte Carlo simulations were used to determine a correction factor with which we scaled the formal uncertainties. The final uncertainties were calculated by quadratically combining the re-scaled formal fit uncertainties with the PSF uncertainties. The data in the overlap region between the four dither positions were averaged. Stars present in the overlap region between the dithered fields in the $H$ and $K$ s observations were used to check
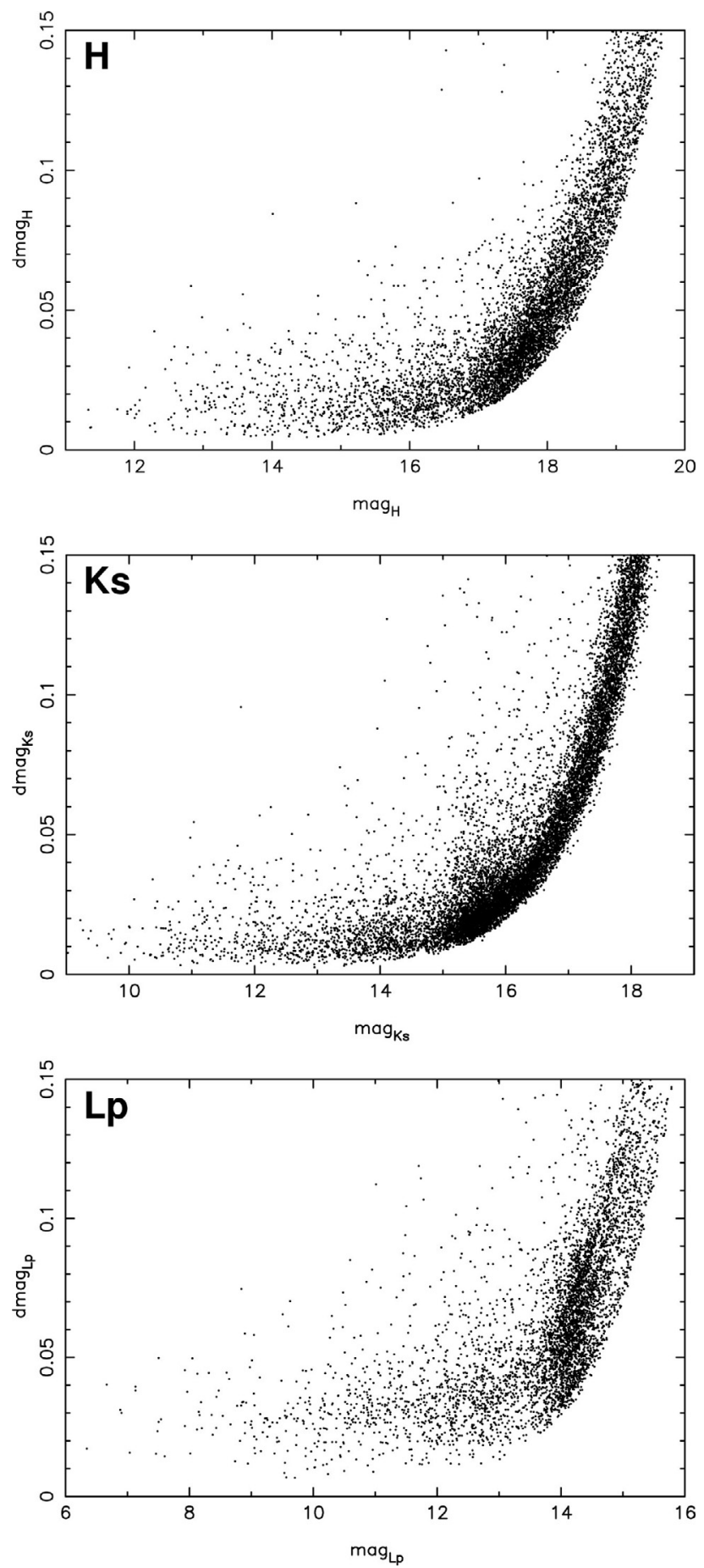

Fig. 3. Statistical photometric uncertainty vs. magnitude for the $H$ (top), Ks- (middle), and $L^{\prime}$ - (bottom) bands.

the photometric accuracy. Photometry on the stars in the overlapping fields shows that the uncertainties estimated by the applied method are correct (see Schoedel, A\&A, 2009, accepted for publication).

Photometry on the $L^{\prime}$ image was performed in a very similar way, except that there was only one combined frame to be examined. The PSF for initial linear deconvolution was extracted from IRS 7. The size of the sub-frames for local PSF fitting was $300 \times 300$ pixel $^{2}$, with a shift of 50 pixels between the frames. Since the $L^{\prime}$ data are very rich in structure as concerns the diffuse emission, we set the back_box to a smaller value (20 pixels). 
Zero points for the NACO instrument for various combinations of cameras, filters, and dichroics (that split the light between wavefront sensor and camera) are determined routinely within the ESO instrument calibration plan. Zero points for all filters used in this work and for the corresponding setup (camera S27, dichroic N20C80) were determined via observation of a standard star during the same night as the observations: $Z P_{H}=$ $23.64 \pm 0.05, Z P_{K \mathrm{~s}}=22.85 \pm 0.05$, and $Z P_{L^{\prime}}=22.38 \pm 0.15$. Additional uncertainty of the zero point is caused by the uncertainty of the PSF that is used for deconvolution. From experiments (choosing different PSF sizes) we estimate this additional uncertainty to $\lesssim 0.03 \mathrm{mag}$. We combine this additional uncertainty quadratically with the previous uncertainties and obtain final zero point uncertainties of $\Delta Z P_{H}=0.06, \Delta Z P_{K \mathrm{~s}}=0.06$, and $\Delta Z P_{L^{\prime}}=0.15$. The annual mean and standard deviation of the zero points corresponding to the year and the setups used for the observations in this work are $Z P_{H \text {,mean,2006 }}=23.61 \pm 0.10$, $Z P_{K \text { s,mean,2006 }}=22.82 \pm 0.07$, and $Z P_{L^{\prime} \text {, mean, } 2008}=22.25 \pm 0.17$. This agrees well with the zero points and corresponding $1 \sigma$ used for the photometry in this work.

Plots of photometric uncertainty vs. magnitude for all point sources discovered in the individual bands are shown in Fig. 3. We combined the photometric results to a final list, requiring that each star must be detected in at least two different bands. The final lists of detected point sources was aligned with the coordinate system of the $K$ s-observations. The full list of all stars and their fluxes in the different bands is given in Table A.2, available online.

Because of the non-standard photometric methodology applied here (deconvolution and extraction of local PSFs) it was not straightforward to determine incompleteness due to crowding via the standard method of inserting and recovering artifical stars. The main difficulty here is the change of the PSF across the FOV. We therefore estimated completeness directly from the data using the methodology suggested by Eisenhauer et al. (1998) and further described in Harayama et al. (2008). In brief, it consists of deriving a critical distance for each possible magnitude difference between two given stars. A star closer to a brighter one than the critical distance will not be detected. Once the critical distance is known for all relevant magnitude differences, completeness maps can be created. Table 2 gives the completeness of our data for different magnitudes and bands. Some of the assumptions of the methodology suggested by Eisenhauer et al. (1998) may be violated in our case, particularly becase the stellar density in the GC FOV changes strongly because of effects of extinction and cluster structure. For this reason we estimated the critical distances in various ways, e.g. by analysing different parts of the FOV. In Table 2 we therefore give two values for estimated completeness, a high and a low one, for each magnitude and band. Fortunately it turns out (see following section) that completeness correction does not have any significant influence on our results.

\section{Constraining extinction with the red clump}

Color-magnitude diagrams of our data are shown in Fig. 4. The densely populated area around $K \mathrm{~s} \approx 15.6$ and $L^{\prime} \approx 14.2$ is due to red clump (RC) stars, which are the equivalent to horizontal branch stars in metal-rich populations. The luminosities and colors of RC stars are narrowly distributed and are only weakly dependent on age and metallicity, particularly for clusters older than 1.6 Gyr (e.g., Castellani et al. 1992; Alves 2000; Grocholski \& Sarajedini 2002).
Table 2. Completeness levels in percent for given bands and magnitudes.

\begin{tabular}{lccc}
\hline \hline mag & $H$ & $K \mathrm{~s}$ & $L^{\prime}$ \\
\hline 12 & $100-100^{a}$ & $98-100^{a}$ & $93-96^{a}$ \\
13 & $100-100$ & $96-98$ & $84-91$ \\
14 & $99-99$ & $91-97$ & $64-77$ \\
15 & $97-99$ & $83-92$ & $54-69$ \\
16 & $93-97$ & $59-82$ & $42-58$ \\
17 & $83-91$ & $47-70$ & n.a. \\
18 & $66-82$ & $28-50$ & n.a. \\
19 & $50-70$ & n.a. & n.a. \\
\hline
\end{tabular}

Notes. ${ }^{(a)}$ A high and a low value of estimated completeness are given for each combination of band and magnitude.

The completeness corrected luminosity functions (LFs) in the $H, K$ s, and $L^{\prime}$ bands are shown in the bottom panel of Fig. 4. The RC feature is clearly visible as a bump in all LFs. We fitted all LFs with the combination of exponentials plus Gaussians. The locations of the peaks of the $\mathrm{RC}$ as derived from these fits are $H_{\mathrm{RC}}=17.60 \pm 0.04(17.58 \pm 0.016), K \mathrm{~s}_{\mathrm{RC}}=15.59 \pm$ $0.04(15.57 \pm 0.009)$, and $L_{\mathrm{RC}}^{\prime}=14.25 \pm 0.03(14.22 \pm 0.008)$. Values in brackets are the results obtained if no crowding completeness correction is applied. The uncertainties of the fits are smaller than the uncertainty of the absolute photometric calibration ( $0.06 \mathrm{mag}$ for $H$ and $K \mathrm{~s}$, and $0.15 \mathrm{mag}$ for $\left.L^{\prime}\right)$. In the following analysis we will use the numbers based on the completeness corrected LFs. We note however, that this crowding completeness corection has a negligible effect, of the order $10 \%$ of the uncertainty, on the final results. We attribute this to two reasons: (a) the RC is located in a magnitude range where completeness is fairly high; (b) the dominating uncertainty is the one of the absolute photometric calibration. That incompleteness due to crowding is not a serious issue can also be seen when comparing the KsLF from this paper with the one shown in Fig. 10 of Schödel et al. (2007a). A different, slightly deeper data set was used for the latter work, but we can see that the RC bump in the $K \mathrm{sLF}$ is negligibly affected by incompleteness. Note, however, that absolute calibration was not a goal of Schödel et al. (2007a), therefore, there RC peak is shifted by $\sim 0.3$ mag to brighter magnitudes. Finally, we may ask whether the strong extinction toward the GC may affect the RC peak in the LFs of the different filters and thus bias the peak toward brighter values. RC stars are, however, well within the sensitivity of the observing setup used, at all bands. For example, an isolated $K \mathrm{~s}=20$ source would be detected with NACO and the settings used for this work at a level of $\sim 10 \sigma$. The only incompleteness that affects us here is the one due to crowding, which has fortunately a negligible effect on our results.

Since RC stars can be found in large numbers toward the Galactic bulge and center, they are highly useful for studying Galactic extinction and structure (e.g., Wozniak \& Stanek 1996; Udalski 2003; Sumi 2004; Nishiyama et al. 2005, 2006a,b). The absolute magnitude of RC stars has been estimated to $M_{K}=-1.54 \pm 0.04$ (in the 2MASS system) by Groenewegen (2008) and to $M_{K}=-1.61 \pm 0.03$ by Alves (2000). Both values are based on the Hipparcos catalogue. Here we adopt the more recent value for $M_{K}$. The difference between the $K$ and $K$ s-magnitude is $\approx 0.01 \mathrm{mag}$ (see discussion in Nishiyama et al. 2006b). Nishiyama et al. (2006b) estimated the distance to the GC based on photometry of RC stars, calculating the distance modulus as

$(m-M)_{0}=K \mathrm{~s}_{\mathrm{RC}, \text { intr. }}-M_{K \mathrm{~s}}+\Delta M_{K}$, 

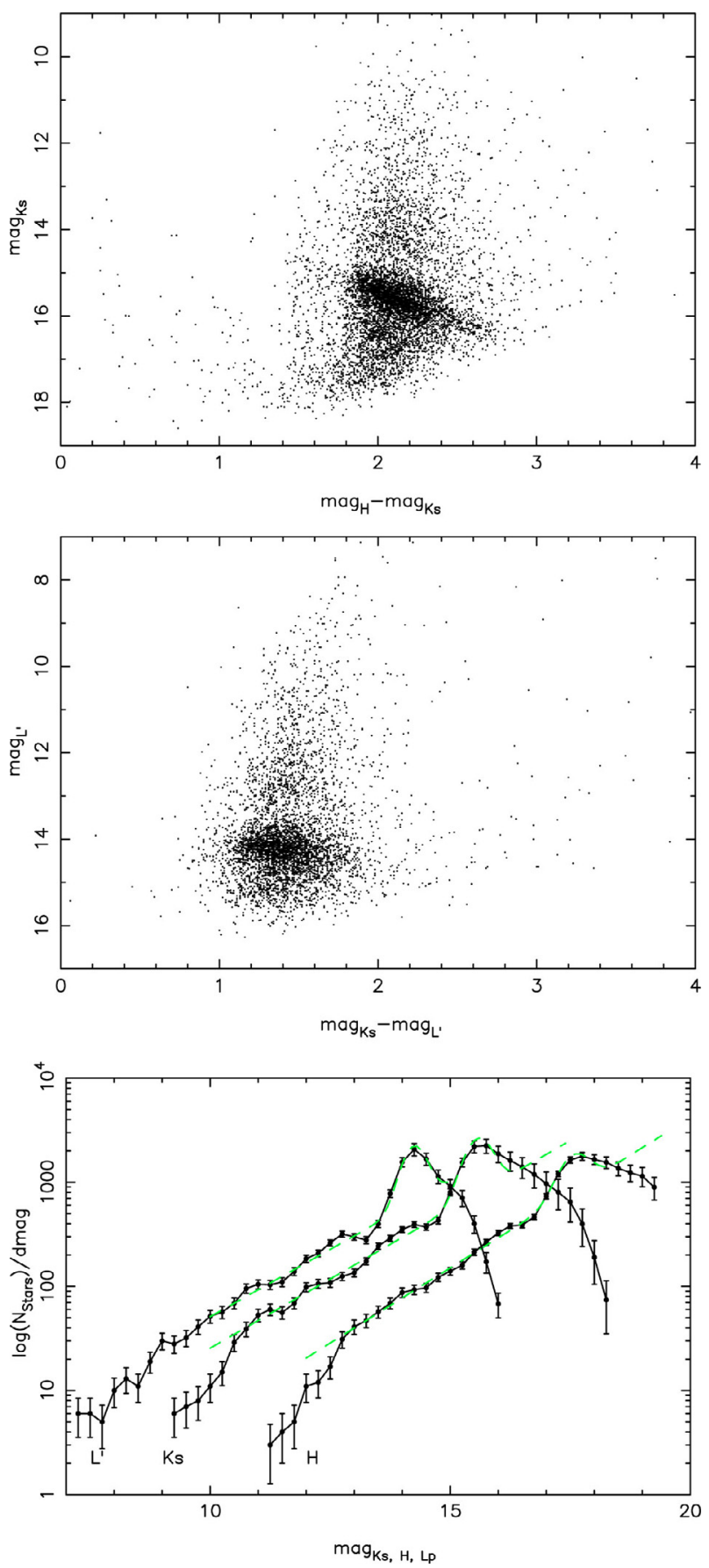

Fig. 4. Color-magnitude diagrams, with $K$ s vs. $H-K$ s shown in the top panel and $L^{\prime}$ vs. $K \mathrm{~s}-L^{\prime}$ in the middle one. Bottom: Completeness (due to crowding) corrected $L^{\prime}, K \mathrm{~s}$, and $H$ luminosity functions. The dashed green lines are fits with an exponential plus a Gaussian.

where $K \mathrm{~s}_{\mathrm{RC} \text {,intr. }}$ is the intrinsic, reddening free magnitude of $\mathrm{RC}$ stars, $M_{K \mathrm{~s}}$ is their absolute $K \mathrm{~s}$-magnitude, and $\Delta M_{K}$ the population correction for $M_{K \mathrm{~s}}$. We adopt the same values as Nishiyama et al. (2006b) for $\Delta M_{K}=-0.07$ and use $M_{K \mathrm{~s}}=-1.54$ (see above). By assuming a GC distance, we can use the above equation to estimate the reddening free magnitude of RC stars. By comparing it subsequently with the magnitude of the RC clump peak as inferred from the luminosity function, we can then obtain an estimate of the absolute extinction in $K$ s toward the GC.

Numerous efforts to determine the distance of the GC have been made recently (the cited uncertainties are the quadratically combined statistical and systematic uncertainties given in the respective works): $7.52 \pm 0.36 \mathrm{kpc}$ (bulge red clump stars, Nishiyama et al. 2006b), $8.33 \pm 0.35 \mathrm{kpc}$ (stellar orbit around Sagittarius A*, Gillessen et al. 2009), $8.0 \pm 0.6 \mathrm{kpc}$ (stellar orbit around Sagittarius A*, Ghez et al. 2008), $7.94 \pm 0.45 \mathrm{kpc}$ (Population II Cepheids and RR Lyrae stars Groenewegen et al. 2008), $8.07 \pm 0.35 \mathrm{kpc}$ (proper motion and line-of-sight velocity dispersion of late-type stars in central parsec, Trippe et al. 2008), $8.4 \pm 0.6 \mathrm{kpc}$ (trigonometric parallaxes of masers measured with VLBI, Reid et al. 2009a), 8.24 $\pm 0.43 \mathrm{kpc}$ (Mira stars, Matsunaga et al. 2009), and $7.9 \pm 0.8 \mathrm{kpc}$ (trigonometric parallax of Sgr B2 measured with VLBI, Reid et al. 2009b). These distance estimates are based on different data sets and different methods and can probably be considered largely independent measurements. It appears therefore justified to combine these measurements to a weighted mean of $R_{0}=8.03 \pm 0.15 \mathrm{kpc}$. This corresponds to a distance modulus of $14.52 \pm 0.04$. This simple exercise demonstrates that - under the assumption that these measurements are indeed statistically independent $-R_{0}$ is already known with the surprisingly small uncertainty of just $2 \%$. Using this value we obtain a reddening free magnitude of the RC bump at the GC of $K \mathrm{~s}_{\mathrm{RC} \text {,intr. }}=13.05 \pm 0.09$. All uncertainties have been quadratically combined $\left(\Delta(m-M)_{0}=0.04 \mathrm{mag}, \Delta M_{K \mathrm{~s}}=0.04 \mathrm{mag}\right.$, $\left.\Delta \Delta M_{K}=-0.07 \mathrm{mag}\right)$.

The fit of the RC bump in the KsLF with a Gaussian (plus an exponential to fit the underlying luminosity function) results in $K \mathrm{~s}_{\mathrm{RC}}=15.59 \pm 0.07$. Here, the uncertainties from the fit of the RC peak and from the absolute photometric calibration have been combined quadratically. From the difference with the theoretical reddening free magnitude of the $\mathrm{RC}$ bump, this results in an average extinction of $A_{K \mathrm{~s}}=2.54 \pm 0.12$ toward the GC.

Assuming $H-K \mathrm{~s}=0.07 \pm 0.03$ and $K \mathrm{~s}-L^{\prime}=0.07 \pm$ 0.03 for the RC stars, we obtain in the same way estimates for $A_{H}=4.48 \pm 0.13$ and $A_{L^{\prime}}=1.27 \pm 0.18$. Note that these values depend only on the well-known properties of RC stars and on the GC distance. They are independent of any assumptions on the extinction-law.

Assuming the validity of a power-law for the extinction law between $H$ and $K \mathrm{~s}$ and between $K \mathrm{~s}$ and $L^{\prime}$, respectively (see, e.g., Nishiyama et al. 2009), we can derive from the above measurements the respective power-law indices $\alpha_{H-K \mathrm{~s}}=2.21 \pm 0.24$ and $\alpha_{K \mathrm{~s}-L^{\prime}}=1.34 \pm 0.29$. Here the uncertainties include the uncertainties of the effective wavelengths at the corresponding bands. The effective wavelengths adopted in this work are $\lambda_{\mathrm{eff}, H}=1.677 \pm 0.018 \mu \mathrm{m}, \lambda_{\mathrm{eff}, K \mathrm{~s}}=2.168 \pm 0.012 \mu \mathrm{m}$, and $\lambda_{\text {eff }, L^{\prime}}=3.636 \pm 0.012 \mu \mathrm{m}$. Details on how these effective wavelengths have been calculated are given in Appendix A.

As can be seen in the color-magnitude diagram shown in Fig. 4 (top left) there is a clear dependency of the $K$ s peak magnitude of the $\mathrm{RC}$ feature on $H-K \mathrm{~s}$. Because of the narrow intrinsic distribution of colors and magnitudes of the RC stars we expect this trend to be almost exclusively due to differential extinction. In order to quantify this relation, we extracted the KsLF in narrow ranges of $H-K$ s. The peaks of the RC features were then fitted with a Gaussian. This method is very similar to the one applied by Nishiyama et al. (2006a), who examined the location of the RC feature in different fields toward the GC in order to derive the reddening law. Figure 5 shows the thus measured RC $K$ s peak magnitude vs. $H-K$ s along with a linear fit. Assuming $A_{\lambda} \propto \lambda^{-\alpha_{H-K \mathrm{~s}}}$, the slope of the line gives $\alpha_{H-K \mathrm{~s}}=2.54 \pm 0.15$. This value agrees within the uncertainties with the one derived from the RC bump in the $H$ and $K$ s luminosity functions (see above) and thus serves as a cross-check. However, we consider 
R. Schödel et al.: Peering through the veil: near-infrared photometry and extinction for the Galactic nuclear star cluster

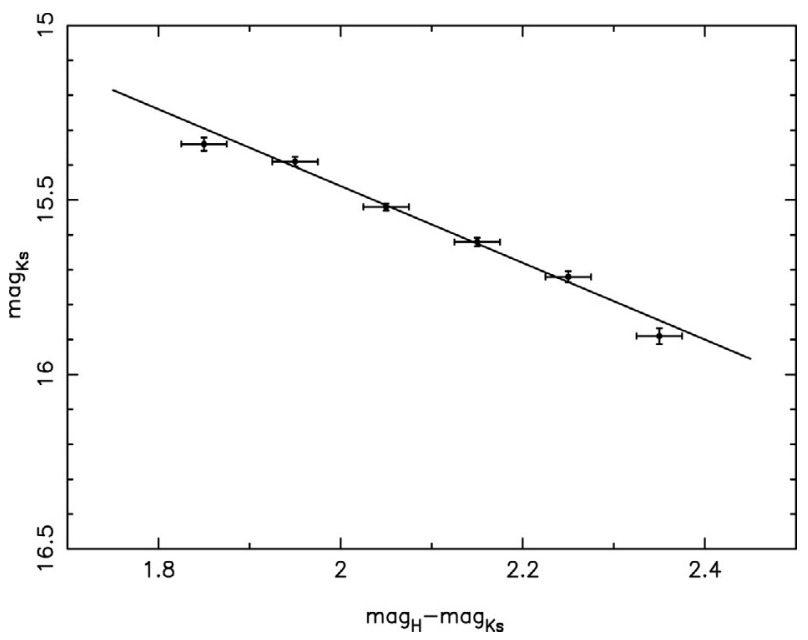

Fig. 5. Peak $K$ s-magnitude vs. $H-K$ s color of the red clump.

the value $\alpha_{H-K \mathrm{~s}}=2.21 \pm 0.24$ derived from the $\mathrm{RC}$ as more reliable (see discussion section).

The $K$ s vs. $H-K$ s color magnitude diagram shows a decreasing completeness with increasing reddening. This is reflected in Fig. 4 by the increasing faint magnitude limit of the cloud of dots toward redder colors. We have seen above that incompleteness does not significantly affect the measured peak of the RC in the KsLF. We have not used any completeness correction for the fit of the RC peak in the color magnitude diagram, but tested its reliability by omitting the two "reddest", i.e. highest $H-K \mathrm{~s}$, data points. The fit then results in $\alpha_{H-K \mathrm{~s}}=2.71 \pm 0.18$. If we additionally omit the bluest data point from the fit, which may be an outlier, we obtain $\alpha_{H-K \mathrm{~s}}=2.45 \pm 0.14$. We conclude that incompleteness due to reddening does not have any significant influence on the value of $\alpha_{H-K \mathrm{~s}}$ derived from the color-magnitude diagram in Fig. 5.

As can be seen in the middle panel of Fig. 4, there is no clear trend of the RC visible in the plot of $L^{\prime}$ vs. $K s-L^{\prime}$. This is mainly because the number of stars measured in $L^{\prime}$ is smaller (smaller FOV) and because the range spanned by the $K \mathrm{~s}-L^{\prime}$ colors is limited due to the weak extinction in $L^{\prime}$. We can therefore not apply this method to determine $\alpha_{K \mathrm{~s}-L^{\prime}}$ (see below for a different method, however).

\section{An extinction map for the GC}

We produced an extinction map for the FOV of the $H$ and $K$ s observations of the GC by using the median of the $H-K$ s colors of the 20 nearest stars at each position. For this purpose we used all stars from our list, but excluded extremely blue $(H-K \mathrm{~s}<1.8)$ or red $(H-K \mathrm{~s}>2.8)$ ones as fore- or background stars. The intrinsic colors of almost all stellar types observable at the GC are similar at these wavelengths. Nevertheless, we tried to improve the estimates by applying a 0th order correction for different intrinsic stellar colors. Stars fainter than $K \mathrm{~s}=14.5$ were assigned $(H-K \mathrm{~s})_{0}=0.07$ and stars brighter than this value were assigned $(H-K s)_{0}=0.2$. Known early-type stars (taken from Buchholz et al. 2009) were assigned $(H-K s)_{0}=-0.03$. In order to assign these intrinsic colors, we used the GC distance, approximate extinction, and the magnitude of the stars to roughly guess their type (see, e.g., Table 3 in Buchholz et al. 2009) and assigned colors from Tables 7.6 and 7.7 in Cox (2000).

In order to check the importance of distinguishing different intrinsic stellar colors we also created a map of $H-K$ s assuming just one uniform intrinsic color, $H-K \mathrm{~s}=0.07$ for all stars. The resulting map looks almost identical to the one including the more refined corrections (the mean difference between the two maps is $\Delta(H-K \mathrm{~s})=0.02 \pm 0.02)$. We conclude that it would be, in principle, acceptable to assume just one common intrinsic color for our entire sample of stars because only $\sim 16 \%$ of our sample of stars are brighter than $K \mathrm{~s}=14.5$. The majority are, in fact, red clump stars. Although this shows that distinguishing different types of stars in the 0th order color correction is almost negligible, we nevertheless include this correction in order to avoid bias in regions where certain types of stars may be crowded. For example, the majority of stars within $0.5^{\prime \prime}$ of Sgr A* are of early-type (e.g. Eisenhauer et al. 2005).

The map of color excesses, $E(H-K \mathrm{~s})$, was converted to an extinction map by assuming a power-law extinction-law with an index $\alpha_{H-K \mathrm{~s}}=2.21 \pm 0.24$ for the effective wavelengths of $\lambda_{H}=1.677 \pm 0.018 \mu \mathrm{m}$ and $\lambda_{K \mathrm{~s}}=2.168 \pm 0.012 \mu \mathrm{m}$. The uncertainty of these three parameters combined with the uncertainty of the photometric zero points and of the intrinsic stellar colors results in a combined systematic uncertainty of $\sim 9 \%$ on the calculated values of $A_{K \mathrm{~s}}$. The extinction map based on $H-K$ s colors is shown in the top left panel of Fig. 6. The top right panel of this figure shows the resolution of the extinction map, i.e. the radius at each position within which 20 stars were detected. The statistical uncertainty, $R M S / \sqrt{N-1}$ (with $N=20$ ), of the extinction at each point in the map is shown in the bottom right panel of Fig. 6. It is $<0.1 \mathrm{mag}$ for the entire map. As a cross-check, we also created an extinction map based on $K s-L^{\prime}$ colors, in a completely analogous way. It is presented in the bottom left panel of Fig. 6 and shows the same patterns as the extinction map based on $H-K$ s colors. Note that details will be more reliable in the map based on $H-K$ s because of the higher point source density measured in these bands. Based on the assumption that the extinction law between $K \mathrm{~s}$ and $L^{\prime}$ is a power-law, we determined the corresponding power-law index, $\alpha_{K \mathrm{~s}-L^{\prime}}$, by requiring that the average $A_{K \mathrm{~s}}$ must be the same, based on $H-K \mathrm{~s}$ or $K \mathrm{~s}-L^{\prime}$ colors. A value of $\alpha_{K \mathrm{~s}-L^{\prime}}=1.35$ has been obtained, with a statistical uncertainty of 0.12 and a systematic uncertainty - due to uncertainties in the effective wavelengths and overall photometric calibration - of 0.29 . This value of $\alpha_{K \mathrm{~s}-L^{\prime}}$ agrees very well with the one derived from the location of the RC bump in the $K \mathrm{~s}$ and $L^{\prime}$ luminosity functions.

A histogram of the values of $A_{K \mathrm{~s}}$ measured based on the $H-K$ s colors is shown in Fig. 7. The histogram is fit well by a Gaussian with a mean value of $A_{K \text { s, mean }}=2.74$ mag and a standard deviation of $0.30 \mathrm{mag}$.

The extinction map was used to produce a differential extinction corrected color magnitude diagram of $K \mathrm{~s}$ vs. $H-K \mathrm{~s}$. It is shown in Fig. 8, after exluding all stars with $H-K$ s $<1.8$ as foreground and $H-K \mathrm{~s}>2.8$ as background stars. It can be seen how the extinction correction significantly reduces the scatter in the diagram (compare with the uncorrected diagram in the upper left panel of Fig. 4). The red clump is very well defined and the giant sequence can be perceived clearly to the right of the dashed line, which indicates the median $H-K$ s color. Stars on the left of the dashed line are expected to be either foreground or early-type stars. Known early-type stars (Buchholz et al. 2009) are marked with blue circles. They provide a sanity check on the reliability of identifying early-type stars via extinction corrected $H-K \mathrm{~s}$ colors. At $K \mathrm{~s}<14$ about $65 \%$ of the known early-type stars are identified correctly. How high is the probability to misidentify late-type stars as early-type with this method? Since the FOV of our work is larger than in comparable work, we limit the analysis for investigating this question to the region within $12^{\prime \prime}$ of 

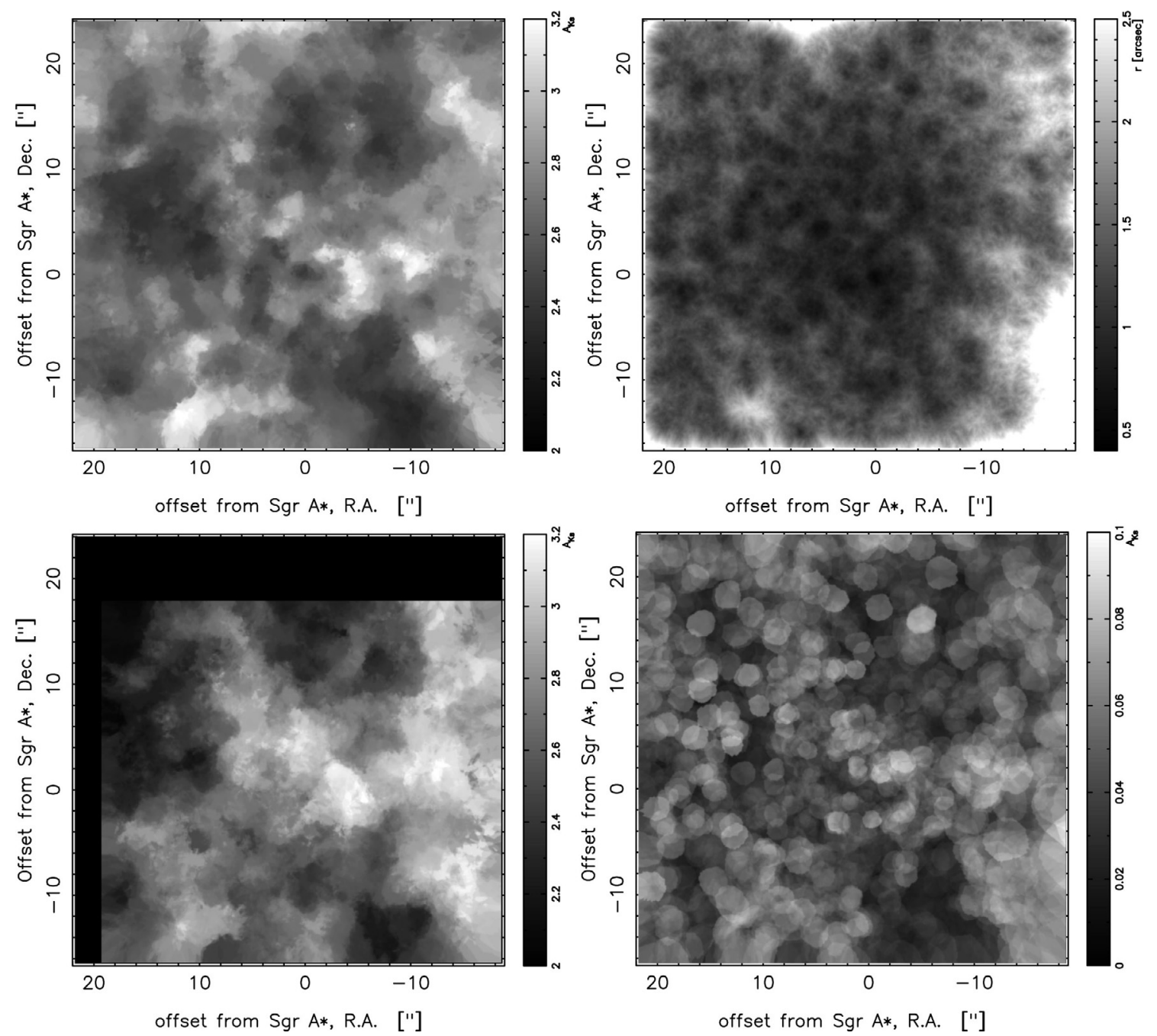

Fig. 6. Top left: map of $A_{K \mathrm{~s}}$ for the GC derived from $H-K$ s colors of all stars detected in these bands, after exclusion of fore- and background stars. Top right: map of the resolution of the extinction, i.e. the radius in arcseconds at each position within which the 20 stars for the given measurement were detected. Bottom left: map of $A_{K \mathrm{~s}}$ for the $\mathrm{GC}$ derived from $K \mathrm{~s}-L^{\prime}$ colors. Bottom right: map of the statistical uncertainty of $A_{K s}$, corresponding to the extinction map shown top left.

Sgr A* and to magnitudes brighter than $K \mathrm{~s}=14$. With these limits we can reasonably assume that the number of known and correctly classified stars (as early- or late-type) from literature is close to $100 \%$. Within $12^{\prime \prime}$ of $\operatorname{Sgr} \mathrm{A}^{*}$ we find that about $30 \%$ of the stars brighter than $K \mathrm{~s}=14$ identified by the extinction corrected $H-K$ s colors may be erroneous identifications. Potential important sources of error are, for example, photometric errors and inaccuracies of the extinction map because of variability at scales below the resolution of our extinction map or because of locally different extinction related possibly with individual sources, e.g. due to circumstellar material. Nevertheless, considering the crudenes of the method presented here, a success rate of $\sim 2 / 3$ appears reasonable and suggests that our method may be useful for large-scale surveys in order to identify candidates for massive, young stars in highly extincted areas. At magnitudes $K$ s $>14$ the extinction corrected $H-K$ s criterion is too crude and does not allow to separate early- and late-type stars in our sample. However, future work may provide more accurate colors and extinction values.

We have marked all stars with $K \mathrm{~s}<14$ and a color bluer than the median $H-K$ s (after excluding foreground stars, see above) with crosses in the $K \mathrm{~s}$ image shown in Fig. 1. Many early-type stars reported by Paumard et al. (2006);
Buchholz et al. (2009); Do et al. (2009a) and other authors are correctly identified. Following the error analysis in the preceding paragraph of the order $30 \%$ of the stars may be spurious identifications and a similar number of early-type stars may be missed by this method. Nevertheless, this is the first time that early-type stars at the GC can be identified via broad-band imaging. Even taking into account the large estimated uncertainties, this rather crude method confirms the finding of Buchholz et al. (2009) that there exists a population of early-type stars beyond a projected radius of $0.5 \mathrm{pc}$ from Sagittarius A*.

Finally, we have used the extinction map to produce a $K$ s luminosity function corrected for differential extinction (fore- and background stars excluded, mean extinction $A_{K \mathrm{~s}}=2.74$, no incompleteness correction applied). It is shown in Fig. 9. By comparing the power-law fit (dashed line) with the data, one can estimate that in the shallow observations used in this work (in order to avoid saturating the brightest stars) the completeness drops below $50 \%$ already at $K \mathrm{~s} \approx 17$ (see also Table 2 ).

\section{Discussion}

For comparison of the derived photometry and extinction with other work, it is important to refer to an effective wavelength, 
R. Schödel et al.: Peering through the veil: near-infrared photometry and extinction for the Galactic nuclear star cluster

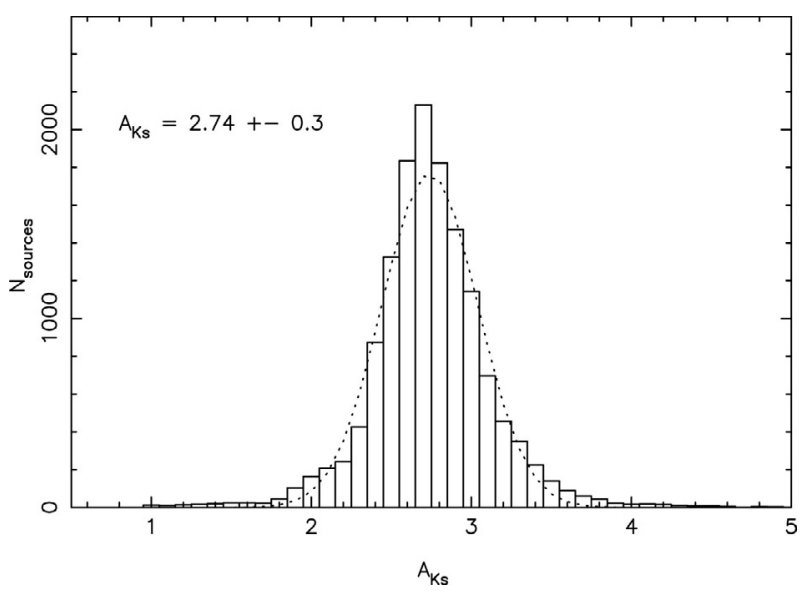

Fig. 7. Histogram of the extinction, $A_{K \mathrm{~s}}$, based on $H-K$ s colors.

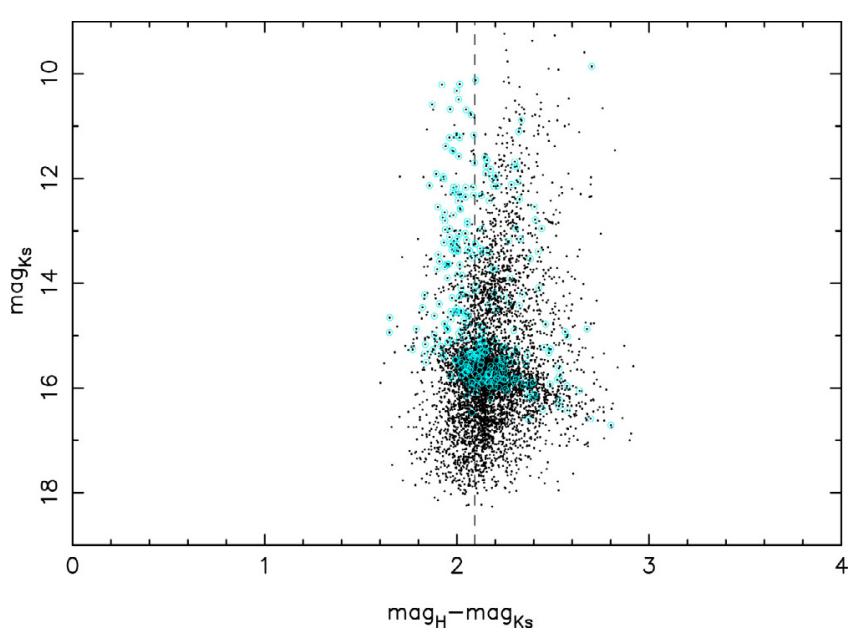

Fig. 8. Differential extinction corrected color-magnitude diagram for the GC (assumed mean extinction is $A_{K s}=2.74$ ). The dashed line indicates the mean $H-K$ s color. Known early-type stars are marked by blue circles.

i.e. to take into account such factors as the SED of the stars, extinction, and the filter transmission curves. The measured value of the exponent of the power-law extinction-law is strongly dependent on the effective wavelength (see, e.g., Nishiyama et al. 2006a; Gosling et al. 2009; Stead \& Hoare 2009). The transmission curves of the NACO filters are available at the ESO website. In order to facilitate comparison of our work, we have calculated the effective wavelengths for the photometry presented here, using Eq. (A3) of Tokunaga \& Vacca (2005).

We have found a significantly steeper extinction law at NIR wavelengths than in earlier work (e.g., Rieke \& Lebofsky 1985; Draine 1989; Mathis 1990, see the latter paper for a review). There exists, however, very good agreement between the values for $\alpha_{H-K \mathrm{~s}}=2.21 \pm 0.24$ (and $\alpha_{H-K \mathrm{~s}}=2.54 \pm 0.15$, respectively) and $\alpha_{K \mathrm{~s}-L^{\prime}}=1.34 \pm 0.29$ (and $\alpha_{K \mathrm{~s}-L^{\prime}}=1.35 \pm 0.31$, respectively) found in this work and the respective values from other recent research. Gosling et al. (2009) report $\alpha_{J-K \mathrm{~s}}=2.64 \pm 0.52$ based on NIR observations of the nuclear bulge. Note that they find variability of this power-law index along the line-of-sight, on scales as small as $5^{\prime \prime}$. We have not examined spatial variability of the power-law index in our work. Since we do not have $J$-band observations available and since the extinction law appears to be clearly different between $J$ and $K$ s and $K$ s and $L^{\prime}$, we cannot determine the extinction-law toward individual stars

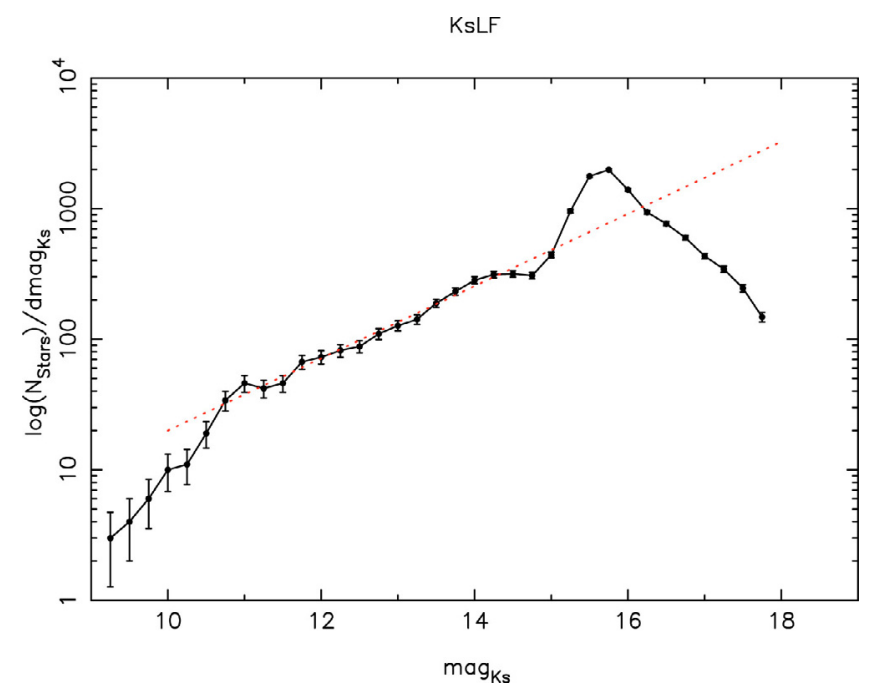

Fig. 9. Ks luminosity function for the GC, fore- and background stars excluded, and corrected for differential extinction. The mean extinction has been set to $A_{K \mathrm{~s}}=2.74$. This luminosity function has not been corrected for completenes. The red dashed line is a power law fit to the stars $11<K \mathrm{~s}<14$. It has a power-law index of $0.27 \pm 0.02$.

or small groups of stars like Gosling et al. (2009). Nishiyama et al. (2009) find $\alpha_{H-K \mathrm{~s}}=2.0$ and $\alpha_{K \mathrm{~s}-3.6}=1.37$, based on NIR observations of the Galactic nuclear bulge. Stead \& Hoare (2009) examined 8 regions from the UKIDDS Galactic Plane Survey and report $\alpha_{\text {NIR }}=2.14 \pm 0.05$. These values agree with our results within the $1 \sigma$ - combined statistical and systematic uncertainties. An exception is the value $\alpha_{H-K s}=2.54 \pm 0.15$ determined from the color dependence of the RC in the $H-K \mathrm{~s}$ vs. $K$ s color-magnitude diagram. Nevertheless, this error lies within the $1 \sigma$ uncertainty of the value $\alpha_{H-K \mathrm{~s}}=2.21 \pm 0.24$ that we have derived in this work from the RC positions in the $K \mathrm{~s}$ and $H$ luminosity functions. We consider the latter value more trustworthy because it is more straightforward to determine (no cutting out of regions from the color-magnitude diagram), is based on large numbers of stars, and agrees very well with the recent works cited above.

The extinction map based on $H-K$ s colors from this work resembles closely the one presented in Buchholz et al. (2009). The overall extinction we find in the present work is, however, lower than the one reported by Buchholz et al. (2009) $A_{K \mathrm{~s}}=2.74 \pm 0.30$ compared to $A_{K \mathrm{~s}}=3.14 \pm 0.43$. The reason for this difference is the assumption of a flatter extinction law (based on Draine 1989) in Buchholz et al. (2009). The extinction map presented by Schödel et al. (2007a) is also similar to the one presented here, but shows a smaller FOV and is also based on a different extinction law. Agreement can also be seen between the extinction map produced in this work and the one presented by Scoville et al. (2003). The map in the latter paper encompasses a much larger region, but is less homogeneous than the one produced here because it is derived from the line emission of ionized gas. Due to the strong radio emission from Sagittarius A* the latter authors could not reliably constrain the extinction in the immediate surroundings of Sagittarius A*. Scoville et al. (2003) used an extinction-law of the form $A_{\lambda} \propto \lambda^{-1.6}$, significantly different from what is found here and in other recent work on this subject.

A caveat may apply to the regions with the strongest extinction. As can be seen in Fig. 1, there are some regions (e.g. at an offset of $\sim+12^{\prime \prime}$ E and $\sim-13^{\prime \prime} S$ of Sagittarius A*) with very 
high extinction and consequently few stars. Extinction may be under-estimated by our method in these patches of extremely high extinction because the number of stars that can be detected behind a strong extinction screen may be very small.

Finally, useful for analyses of the so-called "S-cluster" of stars that are tightly bound to Sagittarius A* and of the NIR emission of Sgr A* itself, we can use the extinction map presented in Fig. 6 to calculate the mean extinction and its standard deviation toward Sagittarius A*. For a circular region with a radius of $0.5^{\prime \prime}$ centered on Sagittarius A* we obtain $A_{H, \mathrm{SgrA} *}=$ $4.35 \pm 0.12, A_{K \mathrm{~s}, \mathrm{SgrA} *}=2.46 \pm 0.03$, and $A_{L^{\prime}, \mathrm{SgrA} *}=1.23 \pm 0.08$. The uncertainty of $A_{K \mathrm{~s} \text {, SgrA* }}$ corresponds to the standard deviation of extinction within the circular aperture. The uncertainties for $A_{H, \mathrm{SgrA} *}$ and $A_{L^{\prime}, \mathrm{SgrA} *}$ include additionally the uncertainties of $\alpha_{H-K \mathrm{~s}}$ and $\alpha_{K \mathrm{~s}-L^{\prime}}$. These extinction values are different from the ones that have typically been used in the analysis of NIR lightcurves from Sgr A*, e.g., $A_{H}=4.3, A_{K}=2.8, A_{L^{\prime}}=1.8$ (Genzel et al. 2003; Eckart et al. 2006), $A_{K}=3.3$ (Do et al. 2009b), or $A_{L^{\prime}}=1.8$ (Ghez et al. 2004). While the impact on emission/accretion models of Sgr A* will probably be minor, we point out that the new extinction values at $K$ s and $L^{\prime}$ imply that the de-reddened emission from $\mathrm{Sgr} \mathrm{A}^{*}$ may be weaker than assumed by a factor $1.4-2.2$ at $K$ s and a factor of $\sim 1.7$ at $L^{\prime}$.

A correct estimate of the extinction is crucial to address one of the long standing issues in the central parsec, namely the apparent incompatibility between the ionizing flux arising from the massive stars and the ionisation of the gas (Najarro et al. 1997; Lutz 1999). Further, these authors found that the population of HeI stars, displaying fairly large luminosities was unusually high. Such incompatibility was recently claimed to be solved by Martins et al. (2007) with the inclusion of line-blanketing in the models, leading to lower bolometric luminosities and lower UV fluxes for the HeI stars. However, we attribute most of the discrepancy between the results of Najarro et al. (1997) and Martins et al. (2007) for the HeI stars to the estimate of $A_{K}$ between both investigations. Indeed, the final $A_{K}$ values computed by Najarro et al. (1997) were 50\% larger than the ones adopted by Martins et al. (2007) from the extinction map of Schödel et al. (2007a). Such difference was translated into roughly one magnitude in luminosity and was responsible for the much lower luminosity of the HeI stars revised by Martins et al. (2007). Similarly, the nature of the "S" stars is also affected. While Martins et al. (2008) adopted $A_{K}=2.25$ for $\mathrm{S} 2$ it turns out that the extinction map they used was calibrated on S2 with an $A_{K}=2.8$ Schödel et al. (2007a). That would have changed S2 from being a dwarf to being a giant. It turns out that the actual value of $A_{K \mathrm{~s}}=2.46$ toward $\mathrm{S} 2$ is not too different from the value assumed (erroneously) by Martins et al. (2008). As we show in this work, and as other similar recent investigation has shown, the solution and key to these problems is that the power-law slope of the NIR extinction law is significantly steeper than what was assumed in earlier work. This leads to lower extinction in the $K$-band, but significantly stronger extinction at shorter wavelengths.

In Fig. 10 we show a color composite image of the stars, detected at $K \mathrm{~s}$, the $A_{K \mathrm{~s}}$ extinction, and the MIR (PAHI) emission (data from Schödel et al. 2007b). It can be seen how the extinction is low in a region running roughly from SW to NE. This creates an apparent high stellar density and therefore elongation of the NSC along the galactic plane (as was also shown by Schödel et al. 2007a). Similarly, the lower extinction to the NW of Sgr A* is responsible for the higher stellar density observed in that region. The strong influence that extinction has on the appearance of the nuclear star cluster can also be nicely seen in Fig. 1 of Scoville et al. (2003). There appears to be no obvious

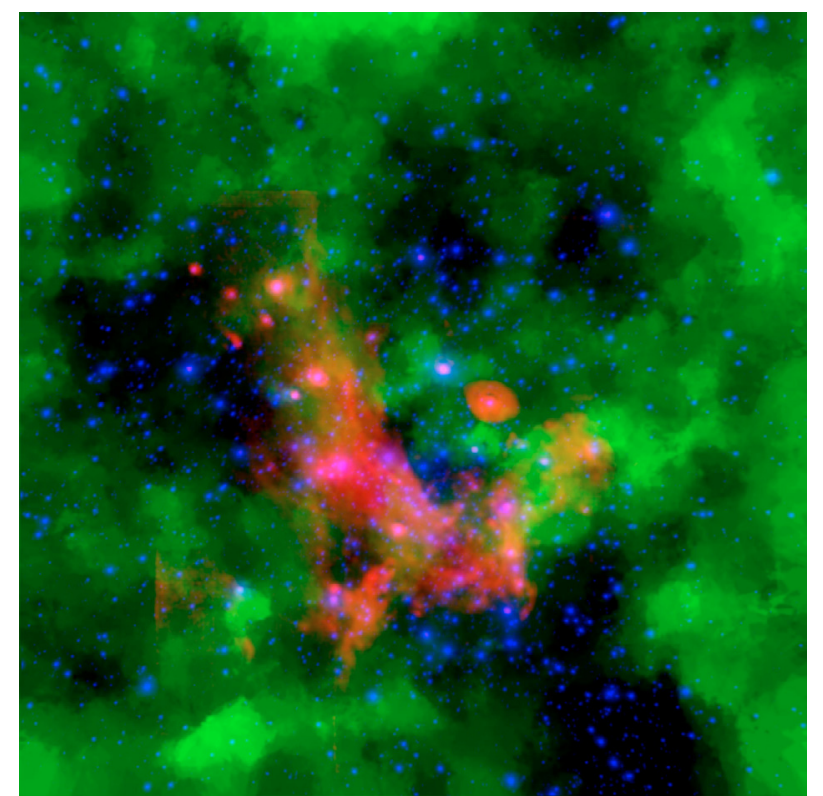

Fig. 10. Ilustration of the relation between the stars detected at $K$ s (blue), the $A_{K \mathrm{~s}}$ extinction (green), and the diffuse emission in the midinfrared (PAHI, red).

relation between the warm dust emitting in the mid-infrared and the extinction. This is no surprise because the surface density of $\mathrm{HI}$ and $\mathrm{H}_{2}$ and the warm dust associated with it in the minispiral is just of the order a few $\times 10^{4} \mathrm{~cm}^{-3}$ (see, e.g., discussion of this topic in Moultaka et al. 2009). The extinction toward the GC is largely produced by clouds in the foreground, with the possible exception of some dust-enshrouded individual objects (Moultaka et al. 2009).

For our analysis we have used a weighted average of recent measurements of the distance to the GC, assuming that all those measurements are independent. We did not investigate this assumption in depth. However, we took into account the systematic uncertainties of the individual measurements when computing the weighted average. As a further simple test we can use sub-samples of the measurements and check the resulting values of $R_{0}$ for consistency. Using only geometrical estimates (Ghez et al. 2008; Trippe et al. 2008; Gillessen et al. 2009; Reid et al. $2009 \mathrm{a}$,b) we obtain $R_{0, \text { geom, } 1}=8.20 \pm 0.22 \mathrm{kpc}$ (emitting the estimate from the S0-2/S2 orbit by Ghez et al. 2008) and $R_{0, \text { geom, } 2}=$ $8.10 \pm 0.26 \mathrm{kpc}$ (emitting the estimate from the S0-2/S2 orbit by Gillessen et al. 2009). Using only measurements based on variable and RC stars, we obtain $R_{0, \text { stars }}=7.85 \pm 0.24 \mathrm{kpc}$. As an additional test we computed the unweighted average of all measurements: $R_{0 \text {,unweighted }}=8.05 \pm 0.28 \mathrm{kpc}$. It agrees within the uncertainties with the weighted average. If we remove the measurement with the strongest apparent deviation $(7.52 \pm 0.36 \mathrm{kpc}$, Nishiyama et al. 2006b), we obtain $R_{0 \text {, unweighted }}=8.12 \pm 0.20 \mathrm{kpc}$. The unweighted averages of the sub-sets of distance measurements considered above agree also well with the weighted results. We therefore believe that the value for $R_{0}=8.03 \pm 0.15 \mathrm{kpc}$ derived in this work and its related uncertainty can be considered reliable.

We believe that future work should focus on deriving the power-law index for the NIR extinction-law toward the GC with higher precision and examine its variability over the central parsecs. This could be achieved, for example, by deep AO imaging using a large number of narrow band filters across the nearinfrared. The main limitation for this kind of work may come 
from the still limited performance of AO systems at short NIR wavelengths ( $J$-band), combined with the long exposure times required by the high extinction. An additional great difficulty, in fact the main source of uncertainty in this work, is the difficulty to obtain precise and accurately calibrated photometry from $\mathrm{AO}$ data (crowding, large seeing foot of PSF, spatial variability of PSF). We have, however, no doubt that there will be significant technical progress in $\mathrm{AO}$ techniques and related data processing software in the next few years.

\section{Summary}

We provide photometry for $\sim 7700$ point sources extracted from adaptive optics observations of the central parsec of the Galactic center in the $K \mathrm{~s}$, and for many of them also in the $H$ and $L^{\prime}$ bands. The data are made publicly available as electronic online material. Adaptive optics photometry has been published previously in some papers. However, we believe that the data presented in this work supersede those earlier results or are complementary to them because of reasons such as: (a) limited field-of-view of previous observations or focus on the central few $0.1 \mathrm{pc}$; (b) isoplanatic effects were not taken properly into account; (c) bias by the application of methods such as LucyRichardson deconvolution (the companion paper to this work, by Schoedel, A\&A, 2009, accepted for publication, explains our photometric method and points out the bias introduced by the Lucy-Richardson method); (d) no transparent explanation of the photometric calibration. This data set is the most comprehensive one published so far.

Based on the photometric results, we analyze extinction toward the central parsec of the GC. Absolute values of extinction in the three examined bands are derived based on the wellknown properties of red clump stars, without the need to measure stellar colors and without having to assume an extinction law. The values obtained are $A_{H}=4.48 \pm 0.13 \mathrm{mag}, A_{K \mathrm{~s}}=$ $2.54 \pm 0.12 \mathrm{mag}$, and $A_{L^{\prime}}=1.27 \pm 0.18 \mathrm{mag}$. This results in $A_{H}: A_{K \mathrm{~s}}: A_{L^{\prime}}=1.76: 1: 0.50$. Assuming the validity of a powerlaw extinction law between $H$ and $K$ s and between $K$ s and $L^{\prime}$, this is equivalent to power-law indices of $\alpha_{H-K \mathrm{~s}}=2.21 \pm 0.24$ and $\alpha_{K \mathrm{~s}-L^{\prime}}=1.34 \pm 0.29$. This is significantly steeper than in earlier work (e.g. Rieke \& Lebofsky 1985; Draine 1989), but agrees with recent studies of extinction toward the nuclear bulge (Nishiyama et al. 2009; Gosling et al. 2009) and other targets in the Galactic plane (Stead \& Hoare 2009). We also confirm the flattening of the extinction law beyond $\sim 3 \mu \mathrm{m}$ (see also Lutz et al. 1996; Indebetouw et al. 2005; Viehmann et al. 2005; Flaherty et al. 2007; Nishiyama et al. 2009).

Based on $H-K$ s colors of the stars, we provide a detailed extinction map for a FOV of $40^{\prime \prime} \times 40^{\prime \prime}(\sim 1.5 \mathrm{pc} \times 1.5 \mathrm{pc})$ centered roughly on Sagittarius A*. The spatial resolution of the map is $\sim 1^{\prime \prime}$. Both statistical and systematic uncertainties of the extinction map are smaller than $10 \%$. The extinction map is made publicaly available as electronic on-line material. We find that extinction varies significantly on arcsecond scales. The mean extinction based on stellar colors is found to be $A_{K \mathrm{~s}}=$ $2.74 \pm 0.30 \mathrm{mag}$, in good agreement with the value of mean extinction based on the RC method. An extinction map based on $K \mathrm{~s}-L^{\prime}$ colors, although less accurate because of lower point source surface density and possible contamination by diffuse emission from dust in the mini-spiral, confirms the patterns seen in the extinction map based on $H-K$ s colors. Requiring the mean extinction between the two maps to be the same, we derive $\alpha_{K \mathrm{~s}-L^{\prime}}=1.35 \pm 0.31$, in excellent agreement with the value
Table A.1. Effective wavelengths in units of $\mu \mathrm{m}$ for the NACO $H, K \mathrm{~s}$, and $L^{\prime}$ filters for blackbodies with different effective temperatures and extinction, $A_{K s}$.

\begin{tabular}{ccccc}
\hline \hline & $2.0^{a}$ & 2.5 & 3.0 & 3.5 \\
\hline$H$ & & & & \\
$3000^{b}$ & 1.674 & 1.681 & 1.688 & 1.695 \\
4700 & 1.669 & 1.677 & 1.684 & 1.691 \\
30000 & 1.664 & 1.672 & 1.680 & 1.687 \\
$K \mathrm{~s}$ & & & & \\
3000 & 2.167 & 2.171 & 2.176 & 2.180 \\
4700 & 2.163 & 2.168 & 2.173 & 2.177 \\
30000 & 2.159 & 2.164 & 2.169 & 2.174 \\
$L^{\prime}$ & & & & \\
3000 & 3.636 & 3.637 & 3.638 & 3.639 \\
4700 & 3.635 & 3.636 & 3.637 & 3.638 \\
30000 & 3.634 & 3.635 & 3.636 & 3.637 \\
\hline
\end{tabular}

Notes. ${ }^{(a)}$ Extinction in the $K$ s band, $A_{K s}{ }^{(b)}$ Effective temperature.

of this parameter derived from the mean magnitudes of the RC bump (see above).

Applying the derived extinction map to the observed $H-K \mathrm{~s}$ colors of the stars, we are able to correct the corresponding color-magnitude diagram. This reduces the scatter in the diagram considerably and makes it possible to distinguish between stars in the giant branch and young, massive stars. We confirm recent findings of the presence of of young, massive stars at projected distances $>0.5 \mathrm{pc}$ from Sagittarius A*.

A minor, but very interesting by-product of this work is a weighted average of recent (years 2006-2009) estimates of $R_{0}$. We find that the distance of the GC is constrained with an uncertainty of just $2 \%: R_{0}=8.03 \pm 0.15 \mathrm{kpc}$.

For a circular region with a radius of $0.5^{\prime \prime}$ centered on Sagittarius A* we obtain $A_{H, \mathrm{SgrA} *}=4.35 \pm 0.12, A_{K \mathrm{~s}, \mathrm{SgrA} *}=$ $2.46 \pm 0.03$, and $A_{L^{\prime}, \mathrm{SgrA} *}=1.23 \pm 0.08$. These values imply that the NIR emission from $\mathrm{Sgr} \mathrm{A}^{*}$ in the $K \mathrm{~s}$ and $L^{\prime}$-bands may be weaker than previously assumed, by factors 1.4-2.2.

We have used the following effective wavelengths in this work: $\lambda_{H}=1.677 \pm 0.018 \mu \mathrm{m}, \lambda_{K \mathrm{~s}}=2.168 \pm 0.012 \mu \mathrm{m}$, and $\lambda_{\mathrm{eff}, L^{\prime}}=3.636 \pm 0.012 \mu \mathrm{m}$. Note that the effective wavelength is a complex function of the transmission functions of the atmosphere, the filter, the optical system, extinction, and stellar type. For discussions of this problem, see, for example, Espinoza et al. (2009) and Stead \& Hoare (2009). The uncertainties cited for the effective wavelengths are based on best-estimates of the variablity of these values for the case of Galactic center observations and the observing conditions and instruments used in this work.

Acknowledgements. R.S. acknowledges the Ramón y Cajal programme of the Spanish Ministerio de Ciencia e Innovación (MICINN) and support by the MICINN project AYA2007-64052. We thank the anonymous referee for his helpful comments. We are grateful to Andrea Ghez and her group at UCLA for providing detailed comments on this work.

\section{Appendix A: Effective wavelength}

We calculated the effective wavelengths of our observations following Eq. (A3) of Tokunaga \& Vacca (2005). The transmission curves for the NACO $H$ and $K$ s filters were downloaded from the instrument web site ${ }^{3}$. Spectra of the atmospheric transmission were downloaded from the Gemini telescope web site ${ }^{4}$. They

\footnotetext{
3 http://www . eso.org/sci/facilities/paranal/

instruments/naco/index.html

4 http://www .gemini.edu/node/10781?q=node/10789
} 
Table A.2. List of detected point sources in the NACO $H, K$ s, and $L^{\prime}$-band observations. We only include the first ten lines of the table in the printed edition of this work.

\begin{tabular}{cccccccccc}
\hline \hline ID & $\begin{array}{c}R_{\text {SgrA* }^{a}} \\
{\left[{ }^{\prime \prime}\right]}\end{array}$ & $\begin{array}{c}\mathrm{RA}^{b} \\
{\left[{ }^{\prime \prime}\right]}\end{array}$ & $\begin{array}{c}\operatorname{Dec}^{c} \\
{\left[{ }^{\prime \prime}\right]}\end{array}$ & $H^{d}$ & $\Delta H^{e}$ & $K \mathrm{~S}^{f}$ & $\Delta K \mathrm{~s}^{g}$ & $L^{\prime h}$ & $\Delta L^{\prime i}$ \\
\hline 1 & 0.028 & 0.009 & -0.027 & 17.50 & 0.05 & 15.7 & 0.04 & $0.0^{k}$ & 0.0 \\
2 & 0.107 & 0.031 & -0.102 & 18.19 & 0.06 & 16.39 & 0.04 & 0 & 0 \\
3 & 0.163 & -0.006 & 0.163 & 16.00 & 0.02 & 14.13 & 0.01 & 12.84 & 0.03 \\
4 & 0.231 & 0.189 & 0.132 & 16.93 & 0.03 & 15 & 0.01 & 0 & 0 \\
5 & 0.245 & -0.231 & -0.081 & 18.69 & 0.07 & 16.75 & 0.05 & 0 & 0 \\
6 & 0.265 & 0.022 & -0.264 & 16.53 & 0.03 & 14.7 & 0.02 & 0 & 0 \\
7 & 0.321 & 0.296 & 0.124 & 16.47 & 0.02 & 14.61 & 0.01 & 0 & 0 \\
8 & 0.347 & -0.274 & 0.213 & 17.45 & 0.03 & 15.66 & 0.09 & 14.35 & 0.06 \\
9 & 0.354 & -0.053 & 0.35 & 17.22 & 0.03 & 15.39 & 0.02 & 0 & 0 \\
10 & 0.363 & 0.02 & -0.363 & 16.23 & 0.03 & 14.12 & 0.01 & 12.6 & 0.03 \\
\hline
\end{tabular}

Notes. ${ }^{(a)}$ Distance from Sgr A* projected on the sky. ${ }^{(b)}$ Offset in right ascension from Sgr A*. Uncertainties in the astrometric positions can be as large as $\sim 0.1^{\prime \prime}$ because high precision astrometry was not at the focus of this work. The conversion to offsets from Sgr A* was done by assuming a pixel scale of $0.027^{\prime \prime}$ per pixel (ESO NACO manual). The camera rotation angle was assumed 0 deg east of north and no camera distortion solution was applied. ${ }^{(c)}$ Offset in declination from Sgr A*. For more information see also footnote ${ }^{b}$. (d) Magnitude in the NACO $H$ band filter. (e) Uncertainty in $H$, obtained by quadratically combining the formal uncertainty from PSF fitting, based on Gaussian read-out noise and Poisson photon noise, and the uncertainty due to the limited knowledge of the PSF. The $1 \sigma$ uncertainty of the zero point, $\Delta Z P_{H}=0.06$, is not included in this column and has to be taken into account when considering absolute photometry. ${ }^{(f)}$ Magnitude in the NACO Ks band filter. ${ }^{(g)}$ Uncertainty in $K$ s. For more information see also footnote ${ }^{e}$. The $1 \sigma$ uncertainty of the zero point is $\Delta Z P_{K \mathrm{~s}}=0.06$. $^{(h)}$ Magnitude in the NACO $L^{\prime}$ band filter. ${ }^{(i)}$ Uncertainty in $L^{\prime}$. For more information see also footnote ${ }^{e}$. The $1 \sigma$ uncertainty of the zero point is $\Delta Z P_{L^{\prime}}=0.15$. ${ }^{(j)} \mathrm{A}$ value of 0.0 in the table indicates that the corresponding measurement is not available.

are based on models with the ATRAN software (Lord 1992). The transmission curves for $3 \mathrm{~mm}$ precipitable water vapor were used because humidity was high during the $H$ and $K$ s observations.

We did not use any particular stellar atmosphere model for the calculations. Instead, simple extincted blackbody models were used. Table A.1 lists the values of the effective wavelength for various values of $A_{K}$ and effective temperatures of the blackbodies. A power-law $A_{K} \propto \lambda^{-\alpha}$ (Nishiyama et al. 2009) was used to calculate the extinction at different wavelengths. We assumed $\alpha_{H-K \mathrm{~s}}=2.22$ for the extinction between $H$ and $K \mathrm{~s}$, and $\alpha_{K \mathrm{~s}-L^{\prime}}=$ 1.33 for the extinction between $K \mathrm{~s}$ and $L^{\prime 5}$. The majority (>90\%) of stars in the images analyzed in this work are of late-type, with a large percentage of RC stars (see, e.g., Buchholz et al. 2009). We therefore adopt as effective wavelengths $\lambda_{\mathrm{eff}, H}=1.677 \mu \mathrm{m}$, $\lambda_{\mathrm{eff}, K \mathrm{~s}}=2.168 \mu \mathrm{m}$, and $\lambda_{\mathrm{eff}, L^{\prime}}=3.636 \mu \mathrm{m}$ for an average extinction of $A_{K S} \approx 2.5$.

The uncertainty introduced by the choice for the amount of precipitable water vapor is low. Choosing $1.6 \mathrm{~mm}$ instead of $3.0 \mathrm{~mm}$ will change the effective wavelengths by $\leq 0.003 \mu \mathrm{m}$ for $H, \leq 0.001 \mu \mathrm{m}$ for $K \mathrm{~s}$, and $\leq 0.01 \mu \mathrm{m}$ for $L^{\prime}$. Changing the power-law index for the extinction curve between $H$ and $K \mathrm{~s}$ to $\alpha_{H-K \mathrm{~s}}=2.0$ (Nishiyama et al. 2009) results in a change of $\leq 0.003 \mu \mathrm{m}$ for $\lambda_{\mathrm{eff}, K \mathrm{~s}}$, and $\leq 0.009 \mu \mathrm{m}$ for $\lambda_{\mathrm{eff}, H}$. Changing the power-law index for the extinction curve between $K \mathrm{~s}$ and $L^{\prime}$ to $\alpha_{K \mathrm{~s}-L^{\prime}}=1.37$ (Nishiyama et al. 2009) results in a change of $<0.001 \mu \mathrm{m}$ for $\lambda_{\mathrm{eff}, L^{\prime}}$. The uncertainty in extinction introduces an uncertainty of $\lesssim 0.015 \mu \mathrm{m}$ for $H, \lesssim 0.01 \mu \mathrm{m}$ for $K \mathrm{~s}$, and $\lesssim 0.002 \mu \mathrm{m}$ for $L^{\prime}$. The unknown effective temperature of the stars introduces uncertainties of $\lesssim 0.005 \mu \mathrm{m}$ for $H, \lesssim 0.006 \mu \mathrm{m}$ for $K \mathrm{~s}$, and $\lesssim 0.001 \mu \mathrm{m}$ for $L^{\prime}$.

\footnotetext{
5 This value of $\alpha_{K s-L^{\prime}}$ is from a preliminary estimate at the beginning of this work and is smaller than the value $\alpha_{K s-L^{\prime}}=1.38$ that we derived in the end. However, the associated change of $\lambda_{\mathrm{eff}, L^{\prime}}$ is minimal, see discussion of the uncertainties in the next paragraph.
}

We estimate the combined $1 \sigma$ uncertainty of the effective wavelengths to $0.018 \mu \mathrm{m}$ for $H, 0.012 \mu \mathrm{m}$ for $K \mathrm{~s}$, and $0.012 \mu \mathrm{m}$ for $L^{\prime}$.

\section{References}

Alves, D. R. 2000, ApJ, 539, 732

Bartko, H., Martins, F., Fritz, T. K., et al. 2009, ApJ, 697, 1741

Böker, T. 2008, J. Phys. Conf. Ser., 131, 012043

Buchholz, R. M., Schödel, R., \& Eckart, A. 2009, A\&A, 499, 483

Castellani, V., Chieffi, A., \& Straniero, O. 1992, ApJS, 78, 517

Cox, A. N. (ed.) 2000, Allen's astrophysical quantities

Devillard, N. 1997, The Messenger, 87, 19

Diolaiti, E., Bendinelli, O., Bonaccini, D., et al. 2000, in SPIE Conf. Ser. 4007, ed. P. L. Wizinowich, 879

Do, T., Ghez, A. M., Morris, M. R., et al. 2009a, ApJ, 703, 1323

Do, T., Ghez, A. M., Morris, M. R., et al. 2009b, ApJ, 691, 1021

Draine, B. T. 1989, in Infrared Spectroscopy in Astronomy, ed. E. Böhm-Vitense, ESA SP-290, 93

Eckart, A., Baganoff, F. K., Schödel, R., et al. 2006, A\&A, 450, 535

Eisenhauer, F., Quirrenbach, A., Zinnecker, H., \& Genzel, R. 1998, ApJ, 498, 278

Eisenhauer, F., Genzel, R., Alexander, T., et al. 2005, ApJ, 628, 246 Espinoza, P., Selman, F. J., \& Melnick, J. 2009, A\&A, 501, 563 Flaherty, K. M., Pipher, J. L., Megeath, S. T., et al. 2007, ApJ, 663, 1069 Genzel, R., Schödel, R., Ott, T., et al. 2003, Nature, 425, 934 Ghez, A. M., Wright, S. A., Matthews, K., et al. 2004, ApJ, 601, L159 Ghez, A. M., Salim, S., Weinberg, N. N., et al. 2008, ApJ, 689, 1044 Gillessen, S., Eisenhauer, F., Trippe, S., et al. 2009, ApJ, 692, 1075 Gosling, A. J., Blundell, K. M., \& Bandyopadhyay, R. 2006, ApJ, 640, L171 Gosling, A. J., Bandyopadhyay, R. M., \& Blundell, K. M. 2009, MNRAS, 394, 2247

Grocholski, A. J., \& Sarajedini, A. 2002, AJ, 123, 1603

Groenewegen, M. A. T. 2008, A\&A, 488, 935

Groenewegen, M. A. T., Udalski, A., \& Bono, G. 2008, A\&A, 481, 441

Harayama, Y., Eisenhauer, F., \& Martins, F. 2008, ApJ, 675, 1319

Indebetouw, R., Mathis, J. S., Babler, B. L., et al. 2005, ApJ, 619, 931 Lord, S. 1992, Tech. Rep.

Lu, J. R., Ghez, A. M., Hornstein, S. D., et al. 2009, ApJ, 690, 1463

Lutz, D. 1999, in The Universe as Seen by ISO, ed. P. Cox, \& M. Kessler, ESA SP-427, 623

Lutz, D., Feuchtgruber, H., Genzel, R., et al. 1996, A\&A, 315, L269

Maness, H., Martins, F., Trippe, S., et al. 2007, ApJ, 669, 1024 
R. Schödel et al.: Peering through the veil: near-infrared photometry and extinction for the Galactic nuclear star cluster

Martins, F., Genzel, R., Hillier, D. J., et al. 2007, A\&A, 468, 233 Martins, F., Gillessen, S., Eisenhauer, F., et al. 2008, ApJ, 672, L119 Mathis, J. S. 1990, ARA\&A, 28, 37

Matsunaga, N., Kawadu, T., Nishiyama, S., et al. 2009, MNRAS, 399, 1709

Moultaka, J., Eckart, A., \& Schödel, R. 2009, ApJ, 703, 1635

Najarro, F., Krabbe, A., Genzel, R., et al. 1997, A\&A, 325, 700

Nishiyama, S., Nagata, T., Baba, D., et al. 2005, ApJ, 621, L105

Nishiyama, S., Nagata, T., Kusakabe, N., et al. 2006a, ApJ, 638, 839

Nishiyama, S., Nagata, T., Sato, S., et al. 2006b, ApJ, 647, 1093

Nishiyama, S., Nagata, T., Tamura, M., et al. 2008, ApJ, 680, 1174

Nishiyama, S., Tamura, M., Hatano, H., et al. 2009, ApJ, 696, 1407

Paumard, T., Genzel, R., Martins, F., et al. 2006, ApJ, 643, 1011

Reid, M. J., Menten, K. M., Zheng, X. W., et al. 2009a, ApJ, 700, 137

Reid, M. J., Menten, K. M., Zheng, X. W., Brunthaler, A., \& Xu, Y. 2009b, ApJ, 705,1548
Rieke, G. H., \& Lebofsky, M. J. 1985, ApJ, 288, 618

Schödel, R. 2010, A\&A, 509, A58

Schödel, R., Eckart, A., Alexander, T., et al. 2007a, A\&A, 469, 125

Schödel, R., Eckart, A., Mužić, K., et al. 2007b, A\&A, 462, L1

Schödel, R., Merritt, D., \& Eckart, A. 2009, A\&A, 502, 91

Scoville, N. Z., Stolovy, S. R., Rieke, M., Christopher, M., \& Yusef-Zadeh, F. 2003, ApJ, 594, 294

Seth, A. C., Blum, R. D., Bastian, N., Caldwell, N., \& Debattista, V. P. 2008, ApJ, 687, 997

Stead, J. J., \& Hoare, M. G. 2009, MNRAS, 400, 731

Sumi, T. 2004, MNRAS, 349, 193

Tokunaga, A. T., \& Vacca, W. D. 2005, PASP, 117, 421

Trippe, S., Gillessen, S., Gerhard, O. E., et al. 2008, A\&A, 492, 419

Udalski, A. 2003, ApJ, 590, 284

Viehmann, T., Eckart, A., Schödel, R., et al. 2005, A\&A, 433, 117

Wozniak, P. R., \& Stanek, K. Z. 1996, ApJ, 464, 233 